\title{
Energy-Efficient Offloading for DNN-based Smart loT Systems in Cloud-Edge Environments
}

\author{
Xing Chen, Member, IEEE, Jianshan Zhang, Bing Lin, Zheyi Chen, Katinka Wolter, Senior Member, IEEE, \\ and Geyong Min, Member, IEEE
}

\begin{abstract}
Deep Neural Networks (DNNs) have become an essential and important supporting technology for smart Internet-of-Things (loT) systems. Due to the high computational costs of large-scale DNNs, it might be infeasible to directly deploy them in energy-constrained loT devices. Through offloading computation-intensive tasks to the cloud or edges, the computation offloading technology offers a feasible solution to execute DNNs. However, energy-efficient offloading for DNN based smart loT systems with deadline constraints in the cloud-edge environments is still an open challenge. To address this challenge, we first design a new system energy consumption model, which takes into account the runtime, switching, and computing energy consumption of all participating servers (from both the cloud and edge) and loT devices. Next, a novel energy-efficient offloading strategy based on a Self-adaptive Particle Swarm Optimization algorithm using the Genetic Algorithm operators (SPSO-GA) is proposed. This new strategy can efficiently make offloading decisions for DNN layers with layer partition operations, which can lessen the encoding dimension and improve the execution time of SPSO-GA. Simulation results demonstrate that the proposed strategy can significantly reduce energy consumption compared to other classic methods.
\end{abstract}

Index Terms-Cloud-edge computing, loT systems, energy-efficient offloading, deep neural networks, particle swarm optimization

\section{INTRODUCTION}

A $S$ the core technology for supporting modern Artificial Intelligence (AI) systems, Deep Neural Networks (DNNs) have made great achievements in smart systems. Due to the powerful analysis capabilities for large-scale data, DNNs are regarded as a promising approach for effectively mining valuable information in the complex environment of Internet-of-Things (IoT) [1]. It has been pointed out that DNNs with more complex and deeper network structures can commonly provide more accurate analysis results [2]. However, more computational resources are consumed when using deeper network structures, which seriously limits the application of large-scale DNNs on energy-constrained IoT devices. One feasible solution is to offload some DNN layers to the remote cloud with sufficient resources [3]. Specifically, DNNs can be divided according

- Xing Chen and Jianshan Zhang are with the College of Mathematics and Computer Science, Fuzhou University, Fuzhou, 350118, China, and with Fujian Provincial Key Laboratory of Network Computing and Intelligent Information Processing, Fuzhou, 350118, China. E-mail: chenxing@fzu.edu.cn, zhangjs0512@163.com.

- Bing Lin is with College of Physics and Energy, Fujian Normal University, Fujian Provincial Key Laboratory of Quantum Manipulation and New Energy Materials, Fuzhou, 350117, China.Fujian Provincial Collaborative Innovation Center for Advanced High-Field Superconducting Materials and Engineering, Fuzhou, 350117, China. Fujian Provincial Collaborative Innovation Center for Optoelectronic Semiconductors and Efficient Devices, Xiamen, 361005, China.E-mail: WheelLX@163.com.

- Zheyi Chen and Geyong Min are with the Department of Computer Science, College of Engineering, Mathematics and Physical Sciences, University of Exeter, Exeter, EX4 4QF, United Kingdom. E-mail: \{zc300, g.min\}@exeter.ac.uk.

- Katinka Wolter is with the Institut für Informatik, Freie Universität Berlin 14195, Germany. E-mail: katinka.wolter@fu-berlin.de.

(Corresponding authors: Bing Lin and Zheyi Chen) to the granularity of DNN layers [4]. For example, complex DNN layers can be offloaded to the cloud for execution, while simple ones are processed locally. However, it might increase the traffic load of core networks and cause high latency due to massive data transmission between IoT devices and the cloud.

Mobile Edge Computing (MEC) would be a promising technology for smart IoT services in response to the above problems. Through offloading computations from the remote cloud to the network edge in proximity to IoT devices, MEC can significantly reduce the data transmission during the preprocessing stage [5]. Moreover, MEC intensifies the processing capacities of mobile networks by deploying computational and storage resources at the network edge. Therefore, it would be an efficient way to alleviate the traffic load of core networks by partitioning DNNs and offloading DNN layers over the cloud, edge, and IoT devices [6].

However, it is still challenging to offload DNN layers while considering energy consumption in the cloud-edge environments. Due to the short battery lifetime of IoT devices, offloading decisions should consider both the deadline constraints of DNN-based smart IoT systems as well as the energy consumption on servers (from the cloud and edge) and IoT devices. When the cloud-edge environments change, the offloading decisions should be adapted. For example, vehicle identification (i.e., an road traffic application) relies on computer vision whose core technology is DNNs. Traffic cameras periodically record the images of onroad vehicles, and these images are processed by the DNNbased applications deployed on the traffic cameras. But these energy-constrained cameras with limited processing capabilities may not complete these computation-intensive 
applications within their deadlines. Therefore, when dealing with the offloading problem of DNN layers, the offloading decisions should be made subject to the consideration of both deadline constraints and energy consumption and adapt to the changes of cloud-edge environments.

In response to these challenges, two research questions are considered: (1) How to design a system model to evaluate the energy consumption of participating equipment including servers and IoT devices? (2) How to develop an energy-efficient offloading strategy to handle the NP-hard offloading problem for DNN-based Smart IoT systems in the cloud-edge environments? To address the above questions, we develop an energy-efficient offloading strategy based on a Self-adaptive Particle Swarm Optimization Algorithm using the Genetic Algorithm operators (SPSO-GA). The major contributions of this work are summarized below:

1) A new system energy consumption model is designed for DNN-based smart IoT systems, which takes into account the runtime, switching, and computing energy consumption of all participating servers (from both the cloud and edge) and IoT devices.

2) An energy-efficient offloading strategy based on SPSO-GA is proposed to reduce the system energy consumption. The proposed strategy can make efficient offloading decisions for DNN layers with layer partition operations, which lessens the encoding dimension and improves the execution time of SPSO-GA.

3) The extensive simulation experiments are conducted. The performance results demonstrate that the proposed strategy can make quick offloading decisions and achieve the superior performance than other classic methods while satisfying deadline constraints.

The remainder of this paper is organized as follows. We review the related work in Section 2. Section 3 introduces the proposed energy-efficient offloading model for DNNbased smart IoT systems in the cloud-edge environments. In Section 4, the proposed SPSO-GA strategy with layer partition is described in detail. Section 5 presents extensive simulation experiments and results to evaluate the performance of our proposed strategy. Section 6 discusses the issues of applicability of SPSO-GA. Finally, Section 7 concludes this paper.

\section{Related Work}

With the increasing complexity of DNN-based smart IoT systems, computation offloading has become an attractive solution for extending the battery lifetime of IoT devices and satisfying the latency requirements of mobile systems [7].

Many research efforts have been devoted to the offloading problems in cloud computing. For example, Altamimi $e t$ al. [8] pointed out that it would be feasible to extend the battery lifetime and capabilities of smartphones by offloading tasks directly to the cloud. They designed a mathematical model to estimate the energy consumption during the task offloading process. Elgazzar et al. [9] proposed a framework of cloud-assisted mobile service provisioning for reliable deliveries. This framework can support online offloading based on the current network and resource status of mobile systems, while meeting user-defined energy constraints.
Fang et al. [10] focused on QoS-aware scheduling of heterogeneous servers for DNN inference workloads in cloud computing. They designed a deep reinforcement learning (DRL) based scheduler to maximize the Quality-of-Service (QoS) including the inference accuracy and response delay. Kumar and Lu [11] argued that IoT systems may not be time-saving or energy-efficient if their computation tasks are offloaded to the cloud directly. Since it would increase the traffic load of core network and cause high latency due to the massive data transmission between IoT devices and the cloud.

MEC is a promising technology to solve the above problem, where the data transmission time in mobile systems can be tremendously reduced by offloading computation from the remote cloud to the edge that is close to IoT devices [12]. Chen et al. [13] used the idea of softwaredefined network (SDN) to study the offloading problem in the ultra-dense networks, aiming to minimize the delay and save the battery life of users' equipments. Chen et al. [14] studied the multi-user offloading problem in a multichannel wireless interference environment and proposed a distributed offloading algorithm based on the game theory, which realized the Nash equilibrium. Ali et al. [15] adopted a deep learning approach and comprehensive mathematical modeling to offload application components to cloudlets, which can achieve high model accuracy and reduce the energy consumption of users' devices.

The aforementioned work focused on the offloading problem without data dependency. However, the data dependency is of important for the offloading problem in MEC [22]. This is because the data dependency exists between each pair of DNN layers. Lo et al. [16] presented a dynamic DNN design technique to manage the workload transmission under the same accuracy requirement in edge computing. They utilized the dynamic network structure and authentic operation ( $\mathrm{AO})$ unit to enhance DNNs, which had a better performance in terms of reducing the amount of workload transmission while achieving the required accuracy. Jeong et al. [17] designed a snapshotbased offloading method for machine learning web systems in edge environments, which was suitable for real DNNbased web systems. Yang et al. [18] designed a framework for runtime system repartitioning in dynamic mobile cloud environments, which can solve the performance degradation problem caused by fluctuant device status and dynamic network. Hu et al. [23] designed a dynamic DNN surgery strategy to partition DNN inference between the cloud and edge at the granularity of the DNN layers. This strategy reduced the system latency and improved throughput by limiting data transmission, but it paid less attention to the offloading problem for DNN layers. Mohammed et al. [19] proposed an adaptive DNN partition scheme and a distributed algorithm based on the matching game method, where the DNN layers were offloaded to fog nodes. Neurosurgeon [4] claimed that excessive latency and energy consumption were generated when uploading massive data of DNNs to the cloud via the wireless network. To cope with this problem, a lightweight scheduler was designed to partition DNN-based applications automatically between end devices and the cloud at the granularity of DNN layers.

Most of the aforementioned work tried to reduce the sys- 
TABLE 1

The comparative analysis of different work ("+": involved; "-": not involved)

\begin{tabular}{|c|c|c|c|c|c|c|c|c|c|c|c|c|c|c|}
\hline \multirow[t]{2}{*}{ Reference } & \multicolumn{3}{|c|}{ Infrastructure } & \multicolumn{2}{|c|}{ Fluctuation } & \multicolumn{2}{|c|}{ Application type } & \multicolumn{2}{|c|}{ Constraint } & \multicolumn{5}{|c|}{ Object } \\
\hline & 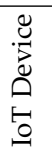 & 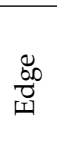 & $\begin{array}{l}\bar{\sigma} \\
\frac{0}{U} \\
\end{array}$ & 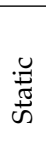 & 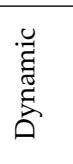 & $\stackrel{\infty}{0}$ & 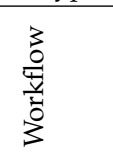 & 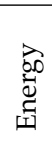 & 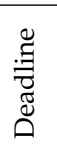 & $\underset{\dot{E}}{\Xi}$ & 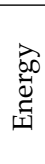 & $\overrightarrow{0}$ & 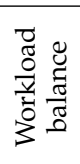 & 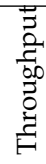 \\
\hline Our work & + & + & + & + & - & - & + & - & + & - & + & - & - & - \\
\hline Hu et al.' [3] & - & + & + & - & + & - & + & - & - & + & - & - & - & + \\
\hline Kang et al. ' [4] & + & - & + & + & - & - & + & - & - & + & + & - & - & + \\
\hline Lin et al. ' [6] & + & + & + & + & - & - & + & - & + & - & - & + & - & - \\
\hline Altamimi et al.' [8] & + & - & + & + & - & + & - & - & - & - & + & - & - & - \\
\hline Elgazzar et al. ' [9] & + & - & + & + & - & + & - & + & - & + & - & - & - & - \\
\hline Fang et al. ' [10] & + & - & + & + & - & - & + & + & - & + & + & - & - & - \\
\hline Chen et al. ' [13] & + & + & - & + & - & + & - & - & - & + & + & - & - & - \\
\hline Chen et al. ' [14] & + & + & - & + & - & + & - & + & - & - & - & - & + & + \\
\hline Ali et al.' [15] & + & + & - & + & - & + & - & + & - & - & - & + & - & - \\
\hline Lo et al. ' [16] & + & + & - & + & - & - & + & - & - & + & + & - & - & - \\
\hline Jeong et al. ' [17] & + & + & - & + & - & - & + & - & - & + & - & - & - & - \\
\hline Yang et al. ' [18] & + & + & + & - & + & - & + & - & - & + & + & - & - & - \\
\hline Mohamed et al. ' [19] & - & + & - & - & + & - & + & - & - & + & - & - & + & - \\
\hline Wu et al. ' [20] & - & + & + & - & + & - & + & - & - & + & + & - & - & - \\
\hline Teerapittayanon et al. ' [21] & + & + & + & + & - & - & + & - & - & - & + & - & - & - \\
\hline
\end{tabular}

tem latency in cloud/edge environments [16], [18], [23], but they did not consider reducing the system energy consumption of offloading DNN layers with deadline constraints. An application partitioning algorithm was presented in [20] for pursuing a trade-off between energy consumption and data transmission in dynamic mobile environments. Teerapittayanon et al. [21] proposed distributed DNNs over the cloud, edge, and IoT devices. They considered the data transmission cost but not the layer execution consumption, while deploying distributed DNNs in the cloud-edge environments. In the previous work [6], a cost-driven offloading scheme was designed for DNN-based smart IoT systems with deadline constraints over the cloud, edge, and IoT devices, where a discrete PSO algorithm was developed to reduce the system cost of executing DNN layers and transferring data. Different from this work, we further consider the energy consumption of each participating server and IoT device, and introduce the layer partition operations into the offloading decision-making process for DNN layers.

The comparative analysis of the previous work has been illustrated in Table 1. In general, most of these work focused on the offloading problem in MEC. However, it is still an open issue to optimize the system energy consumption when offloading DNN layers with deadline constraints in the cloud-edge environments.

\section{Problem Definition and Analysis}

In the cloud-edge environments, DNNs can be deployed in the cloud, edge, and IoT devices. The energy-constrained IoT devices periodically receive terminal information. Some DNN layers are performed on IoT devices while the others are performed in the edge or cloud. Offloading decisions should be made based on the current environment situation, including the server status, network status, and existing tasks, to reduce the system energy consumption by offloading DNN layers while satisfying deadline constraints.
TABLE 2

List of abbreviations

\begin{tabular}{ll}
\hline Abbreviation & Description \\
\hline AI & Artificial Intelligence \\
DNN & Deep Neural Network \\
IoT & Internet-of-Thing \\
MEC & Mobile Edge Computing \\
PSO & Particle Swarm Optimization \\
GA & Genetic Algorithm \\
DRL & Deep Reinforcement Learning \\
QoS & Quality-of-Service \\
AO & Authentic Operation \\
\hline
\end{tabular}

Particularly, when the environment remains unchanged, the offloading decisions will keep the same; and when the environment changes, the offloading decisions need to be adapted for better performance. In this work, we assume that the cloud-edge environments remain constant during a period of time. The major abbreviations and symbols used in this paper are defined in Table 2 and 3, respectively.

\subsection{Problem Definition}

The cloud-edge environments, denoted by $\boldsymbol{C}=\left\{\boldsymbol{C}_{\mathrm{c}}, \boldsymbol{C}_{\mathrm{e}}, \boldsymbol{C}_{\mathrm{d}}\right\}$, consist of the cloud, edge, and IoT devices. Each platform is equipped with some computing nodes (i.e. servers or virtual machines) with different computational capacities. For the clarity of presentation, we use 'server' to represent the computing nodes in different platforms. Thus, there are $n$ servers in the cloud-edge environments, denoted by $\boldsymbol{C}=\left\{s_{1}, s_{2}, \ldots, s_{n}\right\}$, and a server $s_{i}$ is defined as

$$
s_{i}=<p_{i}, t_{i}, c_{i}, \boldsymbol{R}_{i}, \gamma_{i}>
$$

where $p_{i}$ is the computational capacity of $s_{i}$ that is expressed by the CPUs and assumed to be known and static. As this 
study focuses on offloading DNN layers, we assume that the storage capacity of each server can meet the corresponding needs. $t_{i} \in\{0,1,2\}$ represents the type of $s_{i}$, which belongs to IoT devices, edge, and cloud, respectively. $c_{i}$ represents the maximum number of concurrent tasks on $s_{i}$, whose value is equal to the number of cores in $s_{i}$ [13]. When the server $s_{i}$ is fixed, the value of $c_{i}$ will be constant. $\boldsymbol{R}_{i}=\left\{<\zeta_{i}^{1}, \tau_{i}^{1}>,<\zeta_{i}^{2}, \tau_{i}^{2}>, \ldots,<\zeta_{i}^{m}, \tau_{i}^{m}>\right\}$ is the execution status set of $s_{i}$, which expresses the switching frequency of $s_{i}$. Each tuple $\left\langle\zeta_{i}^{j}, \tau_{i}^{j}>\right.$ represents the $j$-th running interval of $s_{i}$ with the start time $\zeta_{i}^{j}$ and end time $\tau_{i}^{j}$. $\gamma_{i}$ represents the energy consumption of $s_{i}$, which is described as

$$
\begin{gathered}
\gamma_{i}=\partial_{i}+\ell_{i}+\eta_{i}, \\
\partial_{i}=\sum_{j=1}^{\left|\boldsymbol{R}_{i}\right|} e_{i}^{r} \cdot\left(\tau_{i}^{j}-\zeta_{i}^{j}\right), \\
\ell_{i}=\sum_{j=1}^{\left|\boldsymbol{R}_{i}\right|} e_{i}^{s}, \\
\eta_{i}=\sum_{j=1}^{\left|\boldsymbol{R}_{i}\right|} \sum_{k=1} e_{i}^{c} \cdot w_{i}^{k} \cdot\left(\tau_{i}^{j}-\zeta_{i}^{j}\right),
\end{gathered}
$$

where $\partial_{i}$ represents the energy consumption of $s_{i}$ running an empty loop, and $e_{i}^{r}$ is the runtime energy consumption of $s_{i}$ per time unit (e.g. second) when it is idle. $e_{i}^{r}$ is assumed to be constant [15]. $\ell_{i}$ represents the switching energy consumption of $s_{i}$, and $e_{i}^{s}$ is the switching energy consumption of $s_{i}$ from power on to power off each time. $\eta_{i}$ represents the computing energy consumption of $s_{i}$, which is related to its workload. $e_{i}^{c}$ is the computing energy consumption of $s_{i}$ per second. $w_{i}^{k}$ is the current workload on $s_{i}$, which may change during the offloading process.

The bandwidth between two servers is defined as

$$
\boldsymbol{B}=\left[\begin{array}{cccc}
b_{1,1} & b_{1,2} & \cdots & b_{1,|\boldsymbol{C}|} \\
b_{2,1} & b_{2,2} & \cdots & b_{2,|\boldsymbol{C}|} \\
\vdots & \vdots & \cdots & \vdots \\
b_{|\boldsymbol{C}|, 1} & b_{|\boldsymbol{C}|, 2} & \cdots & b_{|\boldsymbol{C}|,|\boldsymbol{C}|}
\end{array}\right]
$$

where $b_{i, j}(\forall i, j=1,2, \ldots,|\boldsymbol{C}| ; i \neq j)$ is the bandwidth between $s_{i}$ and $s_{j}$. We assume that there is no ad hoc network, and it is directly unreachable between any two IoT devices [24]. In addition, WiFi has a certain connection range, and thus IoT devices can only connect to the edge servers within its radiation range. Besides, we assume that the bandwidth is not fluctuant.

Each IoT device obtains terminal data periodically, and there are many types of DNNs, denoted by $\boldsymbol{N}=$ $\left\{\boldsymbol{N}_{1}, \boldsymbol{N}_{2}, \ldots, \boldsymbol{N}_{q}\right\}$. A DNN, denoted by $\boldsymbol{N}_{i}=\left(\boldsymbol{L}_{i}, \boldsymbol{H}_{i}, \boldsymbol{D}_{i}\right)$, is regarded as a workflow, where $\boldsymbol{L}_{i}=\left\{l_{i}^{1}, l_{i}^{2}, \ldots, l_{i}^{q}\right\}$ is the set of layers of $\boldsymbol{N}_{i}, \boldsymbol{H}_{i}=\left\{h_{i}^{1,2}, h_{i}^{1,3}, \ldots, h_{i}^{j, k}\right\}$ is the set of data dependencies between layers, and $\boldsymbol{D}_{i}=\left\{d_{i}^{1}, d_{i}^{2}, \ldots, d_{i}^{r}\right\}$ is the set of data transmissions between layers in $\boldsymbol{N}_{i}$. Moreover, the deadline of $\boldsymbol{N}_{i}$ is denoted as $D\left(\boldsymbol{N}_{i}\right)$.

More specifically, a layer $l_{i}^{j}$ in $\boldsymbol{N}_{i}$ is defined as
TABLE 3

Symbol definitions

\begin{tabular}{ll}
\hline Symbol & Definition \\
\hline $\boldsymbol{C}=\left\{\boldsymbol{C}_{\mathrm{c}}, \boldsymbol{C}_{\mathrm{e}}, \boldsymbol{C}_{\mathrm{d}}\right\}$ & $\begin{array}{l}\text { Cloud-edge environments consisting of the } \\
\text { cloud } \boldsymbol{C}_{\mathrm{c}} \text {, edge } \boldsymbol{C}_{\mathrm{e}} \text {, and IoT devices } \boldsymbol{C}_{\mathrm{d}}\end{array}$ \\
$s_{i}$ & Server $i$ \\
$p_{i}$ & Computing capacity of $s_{i}$ \\
$t_{i}$ & Type of $s_{i}$ \\
$c_{i}$ & Maximum number of concurrent tasks on \\
$\boldsymbol{R}_{i}$ & server $s_{i}$ \\
$\zeta_{i}^{j}$ & Execution status set of $s_{i}$ \\
$\tau_{i j}^{j}$ & Start time of the $j^{t h}$ running interval of $s_{i}$ \\
$\gamma_{i}^{j}$ & End time of the $j^{\text {th }}$ running interval of $s_{i}$ \\
$\partial_{i}$ & Energy consumption of $s_{i}$ \\
$e_{i}^{r}$ & Runtime energy consumption of $s_{i}$ \\
$\ell_{i}$ & Runtime energy consumption per second \\
$e_{i}^{s}$ & of $s_{i}$ \\
$\eta_{i}$ & Switching energy consumption of $s_{i}$ \\
$e_{i}^{c}$ & Switching energy consumption per second \\
$w_{i}^{k}$ & of $s_{i}$ each time \\
$b_{i, j}$ & Computing energy consumption of $s_{i}$ \\
$\boldsymbol{N}_{i}=\left(\boldsymbol{L}_{i}, \boldsymbol{H}_{i}, \boldsymbol{D}_{i}\right)$ & Computing energy consumption per sec- \\
$l_{i}^{j}$ & Det $\boldsymbol{H}_{i}$ and data transmission set $\boldsymbol{D}_{i}$ per second \\
$h_{i}^{j, k}$ & Current workload on $s_{i}$ \\
$a_{i}^{j}$ & Layer $j$ in $\boldsymbol{N}_{i}$ \\
$i_{i}^{j}$ & Data dependency between $l_{i}^{j}$ and $l_{i}^{k}$ \\
$o_{i}^{j}$ & Calculation amount of $l_{i}^{j}$ \\
$d_{i}^{j}$ & Input data of $l_{i}^{j}$ \\
$\Omega_{i}^{j}$ & Output data of $l_{i}^{j}$ \\
$t_{t}\left(d_{i}^{j}, s_{k}, s_{r}\right)$ & A data transmission $j$ in $\boldsymbol{N}_{i}$ \\
$t_{i}^{c}$ & Amount of $d_{i}^{j}$ \\
$e_{t}$ & Time for transferring $d_{i}^{j}$ from $s_{k}$ to $s_{r}$ \\
\hline & Completion time of $\boldsymbol{N}_{i}$ \\
& Total energy consumption for executing all \\
DNN layers
\end{tabular}

$$
\begin{gathered}
l_{i}^{j}=<i_{i}^{j}, o_{i}^{j}, a_{i}^{j}>, \\
t_{e}\left(l_{i}^{j}, s_{k}\right)=\frac{a_{i}^{j}}{p_{k}},
\end{gathered}
$$

where $i_{i}^{j}$ and $o_{i}^{j}$ are the input and output data of $l_{i}^{j}$, respectively. $a_{i}^{j}$ is the calculation amount of $l_{i}^{j}$. Therefore, the execution time of $l_{i}^{j}$ on $s_{k}$ is defined as $\left.t_{e}\left(l_{i}^{j}, s_{k}\right)\right)$.

In the data dependency $h_{i}^{j, k}=\left(l_{i}^{j}, l_{i}^{k}\right), l_{i}^{j}$ is the direct precursor of $l_{i}^{k}$, and $l_{i}^{k}$ is the direct successor of $l_{i}^{j}$. A layer can be executed only after all its direct precursors have been completed.

In the data transmission $d_{i}^{j}=<\Omega_{i}^{j}, s_{k}, s_{r}>, \Omega_{i}^{j}$ represents the amount of data transmission, and $s_{k}$ and $s_{r}$ represent the original and final servers storing $d_{i}^{j}$, respectively. Therefore, the data transmission time of $d_{i}^{j}$ from $s_{k}$ to $s_{r}$ is defined as

$$
t_{t}\left(d_{i}^{j}, s_{k}, s_{r}\right)=\frac{\Omega_{i}^{j}}{b_{k, r}} .
$$

Moreover, the proposed offloading strategy is defined as $\boldsymbol{S}=\left(\boldsymbol{C}, \boldsymbol{L}_{i}, \boldsymbol{M}, t_{i}^{c}, e_{t}\right)$, where $\boldsymbol{M}=\left\{\left(l_{i}^{j}, s_{k}\right) \mid l_{i}^{j} \in \boldsymbol{L}_{i}, s_{k} \in\right.$ $C\} \cup\left\{\Phi_{k}\right\}$ represents the mapping set from DNN layers to servers, $\left(l_{i}^{j}, s_{k}\right)$ represents that layer $l_{i}^{j}$ is executed on $s_{k}$, and $\Phi_{k}$ is the execution order of the concurrent layers on $s_{k}$. When the mapping set $M$ is determined, all the datasets 
TABLE 4

Bandwidth between servers

\begin{tabular}{cccc}
\hline$t_{i}$ & & $t_{j}$ & $b_{i, j}\left(\mathrm{MB} \cdot \mathrm{s}^{-1}\right)$ \\
\hline 0 & $\leftrightarrow$ & 1 & 10 \\
0 & $\leftrightarrow$ & 2 & 0.5 \\
1 & $\leftrightarrow$ & 1 & 10 \\
1 & $\leftrightarrow$ & 2 & 0.5 \\
\hline
\end{tabular}

from one server to another is determined accordingly, $t_{i}^{c}$ represents the completion time of $\boldsymbol{N}_{i}$, and $e_{t}$ represents the total energy consumption of all participating servers for executing DNN layers.

$$
\begin{gathered}
t_{i}^{c}=\max _{l_{i}^{j} \in \boldsymbol{L}_{i}}\left\{t^{c}\left(l_{i}^{j}\right)\right\}, \\
e_{t}=\sum_{i=1}^{|C|} \mu \cdot\left(\partial_{i}+\ell_{i}+\eta_{i}\right),
\end{gathered}
$$

where $t^{c}\left(l_{i}^{j}\right)$ represents the completion time of $l_{i}^{j}$, and $\mu$ is the adjustment factor for energy consumption. If the running server belongs to IoT devices, $\mu=1.2$. Otherwise, $\mu=1.0$ [25]. This adjustment design tends to offload more layers to the cloud or edge within their deadlines. Therefore, the objective of the proposed energy-efficient offloading strategy for DNN-based smart IoT systems in the cloudedge environments is formalized as

$$
\begin{aligned}
& \text { Minimize } e_{t}, \\
& \text { subject to } \forall i, t_{i}^{c} \leq D\left(\boldsymbol{N}_{i}\right)
\end{aligned}
$$

\subsection{Problem Analysis}

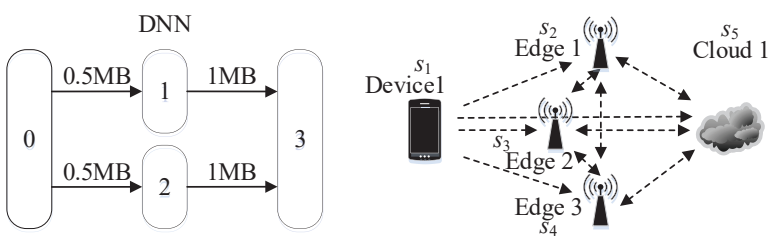

(a) A DNN and the cloud-edge environment. (b) Offloading result by using Greedy.

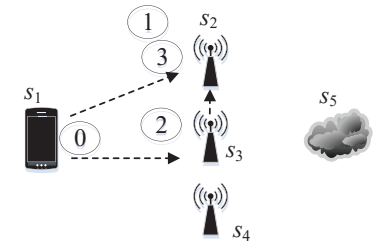

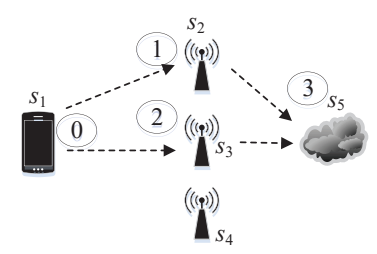

(c) Offloading result by using the optimal strategy.
Fig. 1. An example of energy-efficient offloading for a DNN.

Fig. 1 illustrates an example of energy-efficient offloading for a DNN $\boldsymbol{N}_{i}$ in a cloud-edge environment consisting of six servers, denoted by $\left\{s_{1}, s_{2}, s_{3}, s_{4}, s_{5}, s_{6}\right\} . \boldsymbol{N}_{i}$ contains four layers $\left\{l_{i}^{0}, l_{i}^{1}, l_{i}^{2}, l_{i}^{3}\right\}$ and four data transmissions $\left\{d_{i}^{1}, d_{i}^{2}, d_{i}^{3}, d_{i}^{4}\right\}$ with different data sizes $\{0.5 \mathrm{MB}, 0.5 \mathrm{MB}$, $1 \mathrm{MB}, 1 \mathrm{MB}\}$. The left subgraph in Fig. 1(a) shows the data transmissions between each pair of DNN layers. It should
TABLE 5

Parameter settings about energy consumption for servers

\begin{tabular}{cccc}
\hline Servers & $e_{i}^{r}\left(\mu \mathrm{W} \cdot \mathrm{ms}^{-1}\right)$ & $e_{i}^{c}\left(\mu \mathrm{W} \cdot \mathrm{ms}^{-1} \cdot\right.$ byte $\left.^{-1}\right)$ & $e_{i}^{s}(\mu \mathrm{W})$ \\
\hline$s_{1}$ & 0.1 & 0.1 & 0.3 \\
$s_{2}$ & 0.2 & 0.2 & 0.95 \\
$s_{3}$ & 0.18 & 0.18 & 0.45 \\
$s_{4}$ & 0.22 & 0.22 & 0.65 \\
$s_{5}$ & 0.3 & 0.3 & 0.1 \\
\hline
\end{tabular}

TABLE 6

Calculation amount and execution time of different layers on feasible servers

\begin{tabular}{ccccccc}
\hline & $a_{i}^{j}$ & $s_{1}$ & $s_{2}$ & $s_{3}$ & $s_{4}$ & $s_{5}$ \\
\hline$l_{i}^{0}$ & 1 byte & $1.41 \mathrm{~ms}$ & - & - & - & - \\
$l_{i}^{1}$ & 2 byte & $1.87 \mathrm{~ms}$ & $0.76 \mathrm{~ms}$ & $0.82 \mathrm{~ms}$ & $0.97 \mathrm{~ms}$ & $0.24 \mathrm{~ms}$ \\
$l_{i}^{2}$ & 2.5 byte & $2.78 \mathrm{~ms}$ & $1.51 \mathrm{~ms}$ & $1.42 \mathrm{~ms}$ & $1.67 \mathrm{~ms}$ & $0.82 \mathrm{~ms}$ \\
$l_{i}^{3}$ & 0.5 byte & $3.32 \mathrm{~ms}$ & $2.08 \mathrm{~ms}$ & $1.23 \mathrm{~ms}$ & $2.53 \mathrm{~ms}$ & $0.32 \mathrm{~ms}$ \\
\hline
\end{tabular}

be noted that the input layer $l_{i}^{0}$ must be executed on the IoT device $s_{1}$. The deadline $D\left(\boldsymbol{N}_{i}\right)$ is $5.25 \mathrm{~ms}$. Table 4 shows the bandwidth between servers with different types [6]. Table 5 shows the parameter settings about the energy consumption for different servers. Table 6 shows the calculation amount and execution time of different layers on feasible servers.

Fig. 1(b) depicts the offloading results by using the greedy algorithm [26] without considering the servers in the cloud. This algorithm tends to offload each layer to the corresponding most energy-efficient edge server or IoT device, and thus each layer is offloaded to the server with the lowest computing energy consumption within their deadlines. The completion time $t_{i}^{c}$ of $N_{i}$ is $5.06 \mathrm{~ms}$, and the system energy consumption $e_{t}$ is $5.067 \mu \mathrm{W}$. Fig. 1(c) depicts the optimal offloading results where the completion time $t_{i}^{c}$ is $5.21 \mathrm{~ms}$ and system energy consumption $e_{t}$ is $4.459 \mu \mathrm{W}$ that is less than (about $12.01 \%$ ) the results shown in Fig. 1(b).

\section{Offloading Strategy based on SPSO-GA}

The proposed offloading strategy, denoted by $S=$ $\left(\boldsymbol{C}, \boldsymbol{L}_{i}, \boldsymbol{M}, t_{i}^{c}, e_{t}\right)$, is to explore an optimal mapping $\boldsymbol{M}$ from the DNN layers $\boldsymbol{L}_{i}$ to different types of servers $C$, which aims to reduce the system energy consumption from the perspectives of runtime, switching, and computing, while satisfying the deadline constraints of each DNN. Specifically, a DNN layer can be offloaded to different servers, and an available server can execute many layers from different DNNs. Therefore, it has been proved to be an NP-hard problem to explore the optimal mapping from the layers to servers [27]. The PSO algorithm is be a feasible solution for this problem [6], [28], but it may fall into the local optimum. As for the GA, its local search ability is poor, which results in the low search efficiency in the later stage of evolution [29]. Therefore, these two algorithms cannot well handle the complex offloading problem of DNN layers. In light of the PSO and GA, we propose a new SPSO-GA to explore an optimal offloading strategy for DNN layers in the cloud-edge environments.

\subsection{Layer Partition for DNNs}

The operations of layer partition are to partition consecutive DNN layers into different deployment units. For example, 


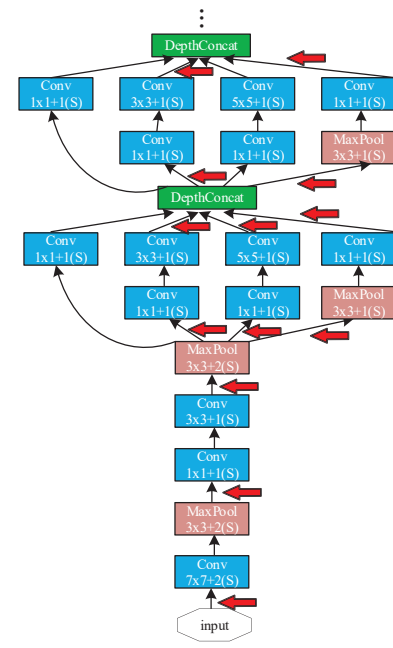

$\lessdot$ partition point
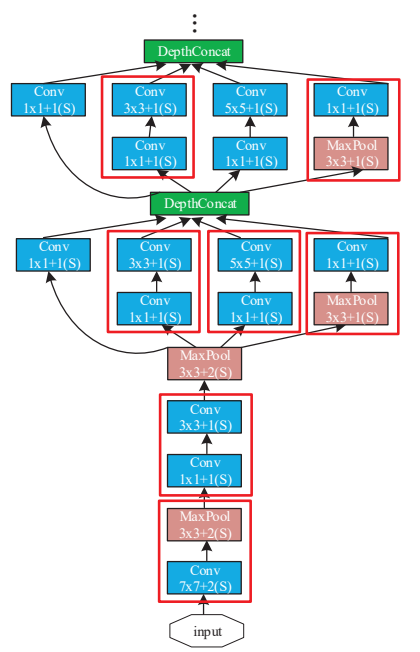

$\square$ deployment unit
Fig. 2. Layer partition for GoogleNet.

as shown in Fig. 2, two DNN layers such as Conv $3 \times 3+1(S)$ and Conv $1 \times 1+1(S)$ are partitioned into a deployment unit, and the DNN layers in this deployment unit would be fitted to be deployed on the same server or IoT device. Algorithm 1 shows the process of layer partition for a DNN, where the input is the original DNN and the output is the DNN with deployment units. It should be noted that the subsequent offloading decisions would be made based on the output DNN. Specifically, different branches in a DNN are first divided into isolated modules (Line 2). For each module, the current layer is initialized as the start layer (Lines 6-8). From the current layer, every two adjacent layers will be checked orderly according to the fitness function defined in Eq. (16) (Lines 10-13). Once a partition point is found, the current layer will be updated to the next layer after this partition point (Lines 14-21). The above process will be repeated until the last two adjacent layers in a DNN are checked. Finally, the layers between every two adjacent partition points are combined into a deployment unit (Lines 23-24).

Under the cloud-edge environments with various computational resources and network connections, there might be different numbers of consecutive DNN layers that are fitted to be deployed together. Basically, with the higher performance ratio $(\lambda)$ between servers and IoT devices and faster data transmission rate $\left(v_{p}\right)$, the offloading tends to happen while the deployment units are constructed by fewer consecutive DNN layers. With the consideration of these factors, the layer partition can be used to find the consecutive DNN layers that are fitted to be deployed on the same server or IoT device and partition them into different deployment units according to the fitness function.

Property 1: Layer partition is able to improve the execution time of the proposed SPSO-GA by partitioning consecutive DNN layers into different deployment units.

In SPSO-GA, the encoding dimension is linearly positive to the number of deployment units in a DNN, and it determines the execution time of the algorithm. Compared to the total number of layers in a DNN, there are fewer deployment units after taking layer partition. Therefore, the layer

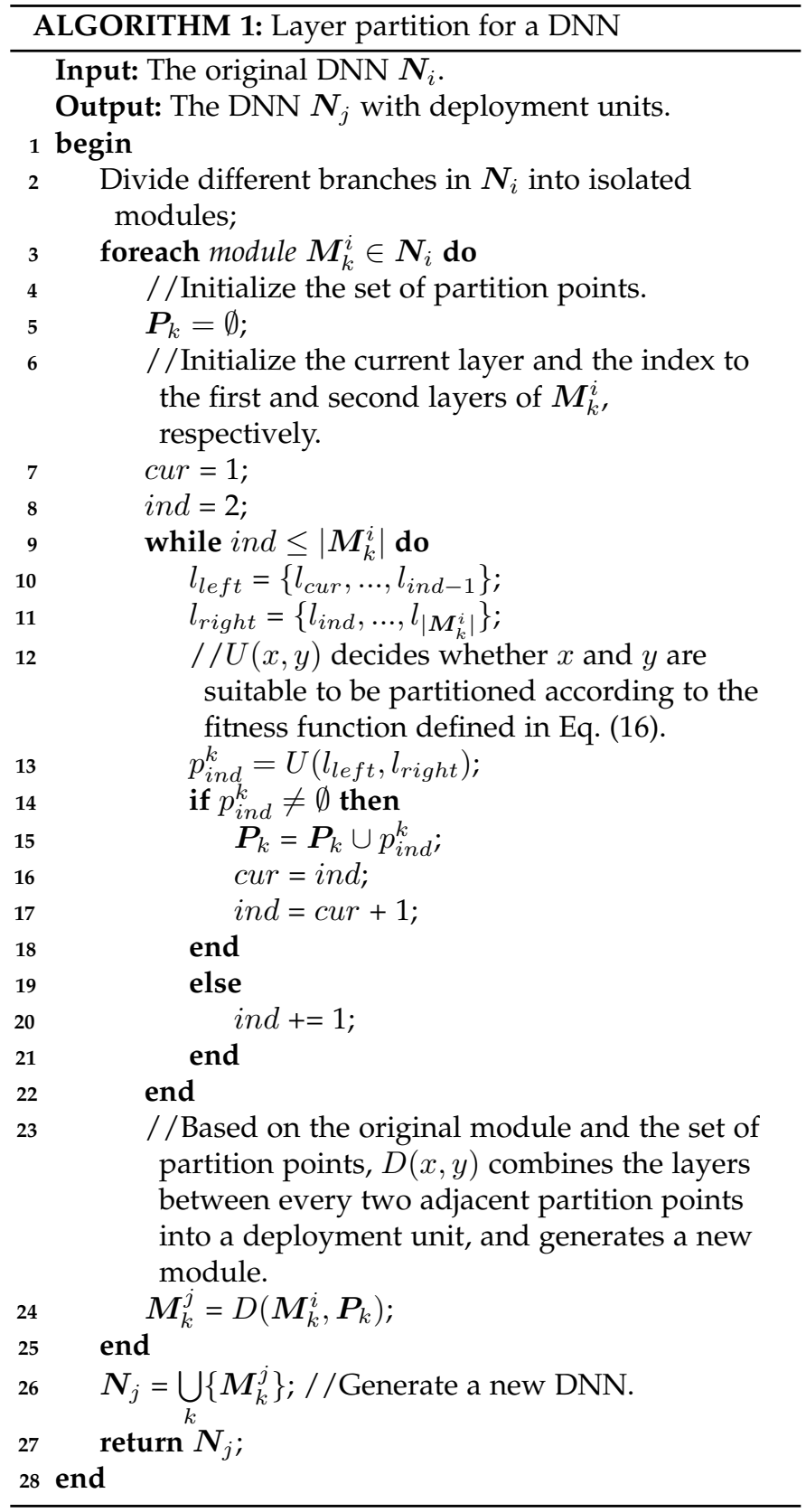

partition can effectively reduce the encoding dimension and improve the execution time of SPSO-GA when considering an offloading problem.

\subsection{Design of SPSO-GA}

In the traditional PSO algorithm, the population commonly contains $n_{p}$ particles, and a particle $Q_{i}^{t}=\left(X_{i}^{t}, V_{i}^{t}\right)$ has its own position, denoted by $X_{i}^{t}=\left(x_{i 1}^{t}, x_{i 2}^{t}, \ldots, x_{i y}^{t}\right)$, and velocity, denoted by $V_{i}^{t}=\left(v_{i 1}^{t}, v_{i 2}^{t}, \ldots, v_{i z}^{t}\right)$, in a continuous space at the $t$-th iteration. The position represents a candidate solution, and the velocity helps each particle to search for better positions. The global best particle in the population at the $t$-th iteration is defined as $G^{t}$, and the personal best particle in its own history at the $t$-th iteration is defined as $P_{i}^{t}$. Thus, the update process for the position and velocity of each particle can be described as 


$$
\begin{gathered}
V_{i}^{t+1}=w \times V_{i}^{t}+c_{1} r_{1}\left(P_{i}^{t}-X_{i}^{t}\right)+c_{2} r_{2}\left(G^{t}-X_{i}^{t}\right), \\
X_{i}^{t+1}=X_{i}^{t}+V_{i}^{t+1},
\end{gathered}
$$

where $w$ is the inertia weight that impacts the convergence of PSO. $c_{1}$ and $c_{2}$ are the acceleration coefficients that represent the cognitive ability of a particle for its personal and global best values, respectively. $r_{1}$ and $r_{2}$ are the random numbers on the interval $[0,1)$ that are used to enhance the random search for an optimal result.

Commonly, the traditional PSO algorithm is used to solve continuous optimization problems. However, the energy-efficient offloading strategy for DNN layers is to explore an optimal mapping from deployment units to different servers, which is a discrete optimization problem. Therefore, the 'problem encoding' and 'population update' parts in SPSO-GA should be further adjusted. In addition, the expression of 'DNN layers' replaces that of 'deployment units' for ease of understanding in this Subsection.

\subsubsection{Problem Encoding}

Problem encoding affects the searchability of the PSO algorithm, which is expected to meet three basic criteria [30] as follows.

Definition 1 (Completeness). Each candidate solution must have at least one corresponding encoded particle.

Definition 2 (Non-redundancy). Each candidate solution has only one corresponding particle.

Definition 3 (Viability). Each candidate solution, corresponding to the encoded particles, is feasible.

The encoding of a constrained optimization problem usually fails to satisfy all three criteria described above. The position of a particle at the $t$-th iteration, denoted by $X_{i}^{t}=\left(x_{i 1}^{t}, x_{i 2}^{t}, \ldots, x_{i y}^{t}\right)$, represents a candidate solution for the optimization problem. Inspired by the work in [31], we use a server-order combination, to encode the energyefficient offloading problem. Fig. 3 illustrates an example of the encoded particle for the offloading results in Fig. 1(c).

\begin{tabular}{|c|c|c|c|c|}
\hline layers & 0 & 1 & 2 & 3 \\
\hline $\begin{array}{l}\text { offloading } \\
\text { location }\end{array}$ & $<1,3\rangle$ & $<2,0>$ & $<3,1>$ & $<5,2>$ \\
\hline
\end{tabular}
Each dimension in a particle is defined as

Fig. 3. An example of the encoded particle for the offloading in Fig. 1(c).

$$
x_{i j}^{t}=<s_{k}, \varphi_{k}>_{i j}^{t},
$$

where $x_{i j}^{t}(j=1,2, \ldots, y)$ is the $j$-th dimension of $X_{i}^{t}$, which represents the offloading decision of the $j$-th layer. The total number of DNN layers is $y . s_{k}$ indicates the execution server for the $j$-th layer, and $\varphi_{k}$ is the execution order of the $j$-th layer on $s_{k}$. $\left(s_{k}, \varphi_{k}\right)_{i j}^{t}$ indicates that the $j$-th layer is executed on $s_{k}$ with the order $\varphi_{k}$ in at the $t$-th iteration. $\varphi_{k}$ is a unique integer on the interval $[0, y)$. When more than two concurrent layers (without data dependency) are offloaded to the same server, the layers with smaller values of orders are executed first.
Property 2: Problem encoding meets the criteria of completeness and non-redundancy, but may fail to meet the viability criterion.

Each offloading solution represents the mappings from layers to servers with a specified order. After making offloading decisions, each DNN layer is allocated to an execution server with the corresponding execution order, which is depicted in the dimension of a particle. Therefore, our encoding method meets the completeness criterion. Moreover, a $2 y$-dimensional particle corresponds to an offloading solution. The $j$-th dimension of a particle represents the execution server $s_{k}$ for the $j$-th layer with the order $\varphi_{k}$. Therefore, an offloading solution only maps to an encoding method, which meets the non-redundancy criterion. However, some particles may be infeasible, which means that the offloading solutions fail to satisfy deadline constraints. For example, a particle of the offloading results in Fig. 1 is $(<1,3>,<2,0>,<3,1>,<4,2>)$, and thus the layers $\left(l_{i}^{0}, l_{i}^{1}, l_{i}^{2}, l_{i}^{3}\right)$ are offloaded to the servers $\left(s_{1}, s_{2}, s_{3}, s_{4}\right)$, respectively. The completion time $t_{i}^{c}$ of $\boldsymbol{N}_{i}$ is $5.51 \mathrm{~ms}$, which exceeds the deadline (i.e. $5.25 \mathrm{~ms}$ ). In this situation the encoding method may fail to meet the viability criterion. Therefore, there are two categories of particles in the problem space as follows.

Definition 4 (Feasible particle). A particle corresponding to a candidate solution meets the deadline constraints of all DNN-based smart IoT systems.

Definition 5 (Infeasible particle). A particle corresponding to a candidate solution fails to meet the deadline constraints of at least one DNN-based smart IoT system.

\subsubsection{Fitness Function}

Fitness function is used to evaluate the performance of particles and to select the global and personal best particles $G^{t}$ and $P_{i}^{t}$, respectively. A particle with a smaller value of the fitness function indicates a better candidate offloading solution. According to our optimization objective, we regard the total system energy consumption as the fitness value of a particle. Since some solutions may exceed the deadlines of DNN-based smart IoT systems, we define the fitness function under three different cases as follows.

Case 1: Both particles are feasible. The one with less total energy consumption would be selected. Therefore, the fitness function is defined as

$$
f\left(X_{i}\right)=e_{t}\left(X_{i}\right)
$$

Case 2: One particle is feasible but another is infeasible. The feasible one would be selected. Therefore, the fitness function is defined as

$$
f\left(X_{i}\right)=\left\{\begin{array}{l}
0, \text { if } \quad \forall i, t_{i}^{c}\left(X_{i}\right) \leq D\left(N_{i}\right) \\
1, \text { else }
\end{array}\right.
$$

Case 3: Both particles are infeasible. The one with less completion time would be selected because it has a greater chance to become feasible after population update. Therefore, the fitness function is defined as

$$
f\left(X_{i}\right)=\max \left\{t_{i}^{c}\left(X_{i}\right)\right\} .
$$




\subsubsection{Population Update}

Eqs. (13) and (14) indicate the particle update for continuous optimization problems, which are affected by three factors: inertia, individual cognition, and social cognition [32]. When it comes to the DNN offloading, the particle update is defined as

$$
X_{i}^{t+1}=F_{3}\left(F_{2}\left(F_{1}\left(X_{i}^{t}, w, r_{1}\right), P_{i}^{t-1}, c_{1}, r_{2}\right), G^{t}, c_{2}, r_{3}\right),
$$

where $F_{1}()$ denotes the mutation operation. $F_{2}()$ and $F_{3}()$ denote the crossover operations. $w$ is the inertia weight. $c_{1}$ and $c_{2}$ are acceleration coefficients. $r_{1}, r_{2}$, and $r_{3}$ are the random numbers on the interval $[0,1)$.

For the inertia part, the velocity of particles is defined as

$$
V_{i}^{t}=F_{1}\left(X_{i}^{t-1}, w, r_{1}\right)=\left\{\begin{array}{cc}
M_{u}\left(X_{i}^{t-1}\right) & r_{1}<w \\
X_{i}^{t-1} & \text { else }
\end{array},\right.
$$

where $M_{u}()$ denotes the mutation operator, and $w$ denotes the mutation probability. Mutation operator randomly selects a location ind in a particle, and changes the corresponding value of the server-order tuple. The new value of server $s_{k}$ is a random integer between 1 and $|C|$, and the corresponding order value is also a random integer between 0 and $y-1$. Since the order value in a particle is unique, the other location with the same order is selected and its order value is changed to the original value of location $i n d$. Fig. 4 depicts the mutation operator, which randomly selects a location $i n d_{1}$ and changes the value of the server-order tuple from $\langle 5,2\rangle$ to $\langle 4,3\rangle$. Next, the order value of the first location is changed to 2 .

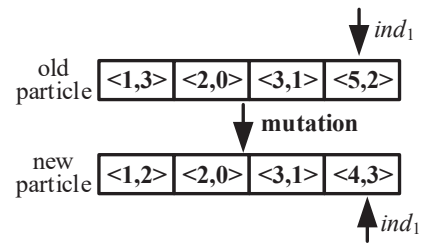

Fig. 4. Mutation operator.

Property 3: The mutation operator may change a particle from feasible to infeasible, and vice versa.

For example, the completion time of the old particle in Fig. 4 is $5.21 \mathrm{~ms}$, and thus the old particle is feasible. After mutation operator, the completion time of the new particle is $5.51 \mathrm{~ms}$, which exceeds its deadline (i.e. $5.25 \mathrm{~ms}$ ). Therefore, the mutation operator may change the old particle from feasible to infeasible. If the positions of the old and new particles are reversed, the mutation operator will change the old particle from infeasible to feasible.

The individual cognition and social cognition in the particle update are formulated as

$$
\begin{gathered}
C_{i}^{t}=F_{2}\left(V_{i}^{t}, P_{i}^{t-1}, c_{1}, r_{2}\right)=\left\{\begin{array}{cc}
C_{p}\left(V_{i}^{t}, P_{i}^{t-1}\right) & r_{2}<c_{1} \\
V_{i}^{t} & \text { else },
\end{array}\right. \\
X_{i}^{t+1}=F_{3}\left(C_{i}^{t}, G^{t}, c_{2}, r_{3}\right)=\left\{\begin{array}{cc}
C_{p}\left(C_{i}^{t}, G^{t}\right) & r_{3}<c_{2} \\
C_{i}^{t} & \text { else }
\end{array}\right.
\end{gathered}
$$

where $c_{1}$ and $c_{2}$ denote the crossover probability of a particle with its personal best solution $P_{i}^{t}$ and global optimal solution $G^{t}$, respectively. $C_{p}()$ denotes the crossover operator. For example, $C_{p}(A, B)$ randomly selects two locations in the particle $A$ and then replaces the server-order segment between these two locations with the same interval in the particle $B$. The order of the generated particle needs to be adjusted. Fig. 5 depicts the crossover operator, which randomly selects the locations $i n d_{1}$ and $i n d_{2}$ in an old particle and replaces the server-order segment between $i n d_{1}$ and $i n d_{2}$ with the same interval in $P_{i}^{t}$ (or $G^{t}$ ). Next, the order value of the 4 th location in the old particle is changed to 2 .

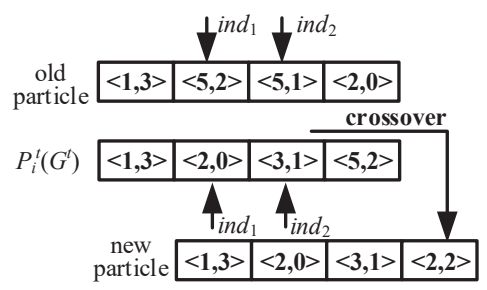

Fig. 5. Crossover operator.

Property 4: The crossover operator may change a particle from infeasible to feasible, and vice versa.

For example, the completion time of the old particle in Fig. 5 is $7.31 \mathrm{~ms}$, which exceeds the deadline (i.e. 5.25 $m s)$. Therefore, the old particle is infeasible. After crossover operator, the completion time of the new particle is $5.06 \mathrm{~ms}$. Therefore, the crossover operator changes the old particle from infeasible to feasible. If the positions of the old and new particles are reversed, and the global optimal particle is $(\langle 1,3\rangle,\langle 4,0\rangle,\langle 2,1\rangle,\langle 5,2\rangle)$, the crossover operator will change the old particle from feasible to infeasible.

\subsubsection{Offloading corresponding a particle}

Algorithm 2 shows the offloading corresponding to a particle. The inputs contain DNNs $N$, all feasible servers $C$, and an encoded particle $X_{i}^{t}$. According to the encoded particle $X_{i}^{t}$, the algorithm first assumes that each layer $l_{i}^{j}$ is offloaded to the server $s_{x(j)}$ with the order $\varphi_{x(j)}$. For $l_{i}^{j}$, its start time $t_{\text {start }}\left(l_{i}^{j}\right)$ is equal to the ready time $t_{\text {ready }}\left(s_{x(j)}\right)$ of $s_{x(j)}$ if it has no parents. Otherwise, the layer cannot start until the data transmission between $s_{x(j)}$ and its parents is completed (Lines 4-13). Next, the end time $t_{\text {end }}\left(l_{i}^{j}\right)$ of $l_{i}^{j}$ is equal to the sum of its execution time $t_{e}\left(l_{i}^{j}, s_{x(j)}\right)$ on $s_{x(j)}$ and start time (Line 14). If $t_{\text {end }}\left(l_{i}^{j}\right)$ exceeds the deadline, the algorithm stops immediately and return $\mathrm{Null}$, which means that this particle is infeasible (Lines 15-17). The next ready time of $s_{x(j)}$ should be calculated until the data transmission between $l_{i}^{j}$ and its children is completed (Lines 18-22). Moreover, the algorithm checks each free interval for all participating servers and shuts down the servers during the free intervals if their runtime energy consumption $\partial_{i}$ is greater than their switching energy consumption $\ell_{i}$ (Lines 24-28). Finally, the system energy consumption $e_{t}$ is calculated based on Eq. (11). 


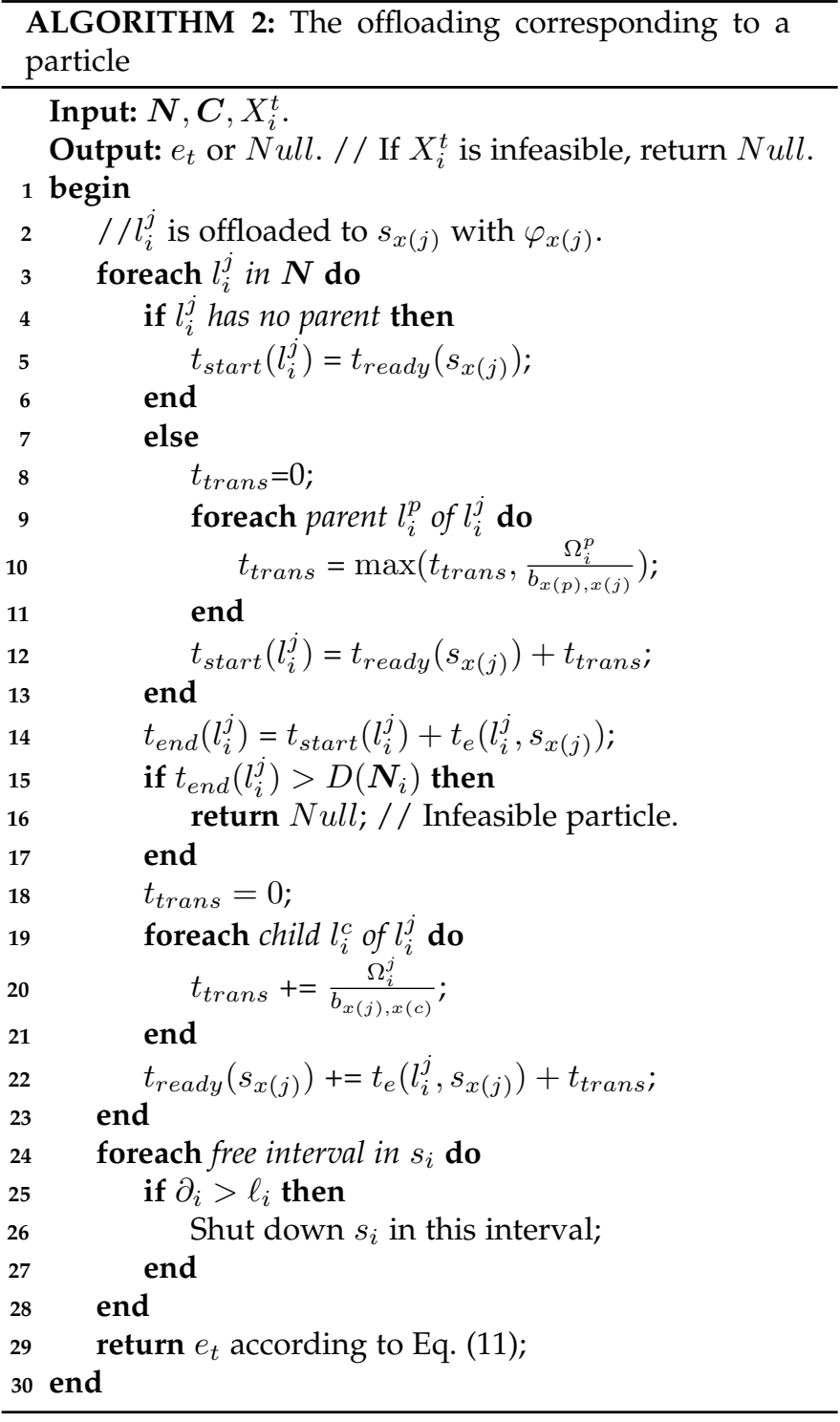

\subsubsection{Parameter Settings}

The inertia weight $w$ may greatly influence the search ability and convergence of the PSO algorithm [33]. The larger value of $w$ corresponds to a stronger global search ability, while the smaller one reflects a better local search ability. To fit in the nonlinear characteristics of energy-efficient offloading for DNN-based smart IoT systems, we design a discrete adjustment method for updating $w$ as

$$
\begin{gathered}
w=w_{u}-\left(w_{u}-w_{d}\right) \times \exp \left(\frac{\Psi}{\Psi-1.01}\right), \\
\Psi=\operatorname{div}\left(G^{t-1}, X^{t-1}\right)=\frac{\sum_{i=1}^{y} z_{i}}{y},
\end{gathered}
$$

where $w_{u}$ and $w_{d}$ denote the maximum and minimum of $w$ during initialization, respectively. $\Psi$ represents the degree of difference between the global optimal solution $G^{t-1}$ and the current candidate solution $X^{t-1} . z_{i}$ denotes a statistical factor. $z_{i}=1$ indicates that the server $s_{k}$ of $G^{t-1}$ and $X^{t-1}$ in the same location is different otherwise is $z_{i}=0$. Therefore, the search ability of the proposed algorithm can be adaptively adjusted based on the difference between the current and the global optimal particles. When the gap between $G^{t-1}$ and $X^{t-1}$ is large, the algorithm has a strong global search ability. On the contrary, the algorithm would enhance its local search ability to explore an optimal solution.

Moreover, the acceleration coefficients $c_{1}$ and $c_{2}$ are used for communications in the population whose settings are referred to the work in [34]. More specifically, $c_{1}^{s}$, and $c_{2}^{s}$ are the start value of $c_{1}$ and $c_{2}$, while $c_{1}^{e}$ and $c_{2}^{e}$ are the end value of $c_{1}$ and $c_{2}$.

\subsubsection{Algorithm Flowchart}

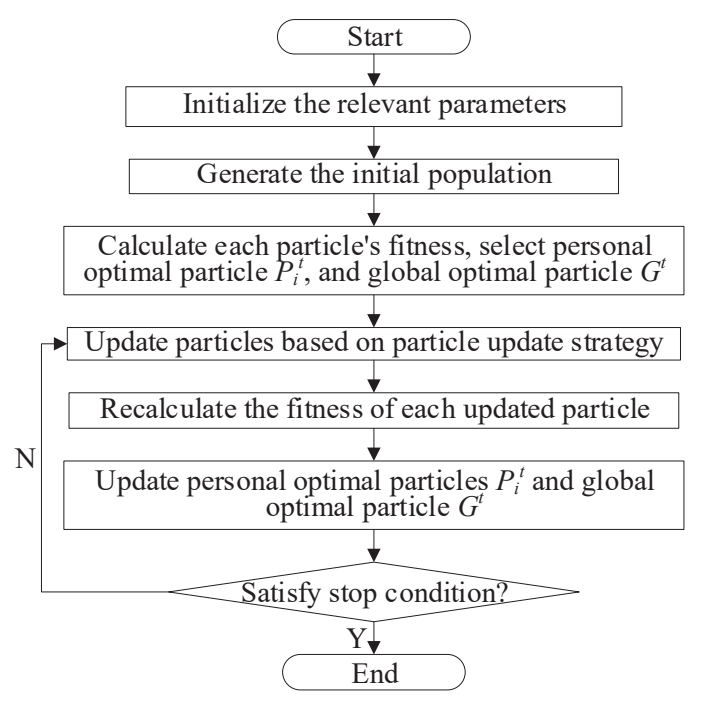

Fig. 6. Algorithm flowchart for SPSO-GA.

Fig. 6 depicts the flowchart of the proposed SPSO-GA, where the detailed process is described as follows.

Step 1: The relevant parameters of SPSO-GA are initialized, including the initial population size $\Gamma$, maximum number of iterations $\Theta$, the maximum inertia weight $w_{u}$, the minimum inertia weight $w_{d}$, the start and end value of acceleration coefficients $c_{1}^{s}, c_{2}^{s}, c_{1}^{e}, c_{2}^{e}$. Next, the initial population is generated randomly.

Step 2: The fitness of each particle is calculated according to Eqs. (16) to (18). Each particle is selected as its own personal optimal solution, and the particle with the best fitness is selected as the global optimal solution in the current generation.

Step 3: Each particle is updated according to Eq. (19), and the fitness of each new particle is recalculated.

Step 4: The personal optimal particle for each particle is updated. The global optimal particle will be updated if there is a better solution than the original one.

Step 5: If the stop condition will be met, the algorithm is determined. Otherwise, the algorithm goes to Step 3.

\section{Performance Evaluation}

To investigate and validate the effectiveness of the proposed SPSO-GA, extensive simulation experiments have been conducted in response to the research questions (RQs) as follows. 
TABLE 7

The related configurations and energy consumption of 15 servers

\begin{tabular}{ccccccc}
\hline Servers & $p_{i}$ & $c_{i}$ & $t_{i}$ & $e_{i}^{r}$ & $e_{i}^{s}$ & $e_{i}^{c}$ \\
\hline$\left\{s_{1}, s_{2}, \ldots, s_{10}\right\}$ & 0.5 & 1 & 0 & 0.1 & 30 & 0.1 \\
$s_{11}$ & 1 & 4 & 1 & 0.2 & 45 & 0.2 \\
$s_{12}$ & 0.8 & 4 & 1 & 0.18 & 45 & 0.18 \\
$s_{13}$ & 1.2 & 4 & 1 & 0.22 & 45 & 0.22 \\
$s_{14}$ & 1.5 & 4 & 1 & 0.27 & 45 & 0.27 \\
$s_{15}$ & 2 & 8 & 2 & 0.3 & 60 & 0.3 \\
\hline
\end{tabular}

The unit of $p_{i}, e_{i}^{r}, e_{i}^{s}, e_{i}^{c}$ is $\mathrm{Hz}, \mu \mathrm{W} \cdot \mathrm{ms}^{-1}, \mu \mathrm{W}, \mu \mathrm{W} \cdot \mathrm{ms}^{-1} \cdot$ byte $^{-1}$, respectively.

- RQ1: Compared with other classic methods, is SPSO-GA able to optimize the system energy consumption more effectively with different deadline constraints? (Section 5.3)

- RQ2: For the same optimization objective, is SPSOGA superior to other classic methods with different workloads? (Section 5.4)

- RQ3: What impact do varying supplies of MEC resources have on the performance of SPSO-GA and other classic methods? (Section 5.5)

- RQ4: Whether the layer partition operations are able to effectively reduce the execution time of SPSO-GA? (Section 5.6)

\subsection{Experimental Settings}

There are four types of DNNs used in the experiments including AlexNet, VGG19, GoogleNet, and ResNet101 [35]. The detailed information about each type of DNN, including the structure, calculation amount of each layer, and data transmissions between layers, which are obtained from actual execution and simulation modeling [36], can be found in our GitHub project ${ }^{1}$. Besides, the deadline constraints for each DNN are needed to check whether an energy-efficient offloading strategy for DNN-based smart IoT systems is feasible or not. Therefore, five different deadline constraints for each DNN are set as

$$
D_{j}\left(\boldsymbol{N}_{i}\right)=\varepsilon_{j} \cdot H\left(\boldsymbol{N}_{i}\right), \varepsilon_{j}=\{1.5,2,3,5,8\},
$$

where $H\left(\boldsymbol{N}_{i}\right)$ is the execution time of $\boldsymbol{N}_{i}$ by using the HEFT algorithm [37], and the values in $\varepsilon_{j}$ are set according to [6].

Furthermore, the cloud-edge environments consist of 15 servers $\left\{s_{1}, s_{2}, \ldots, s_{15}\right\}$, which are divided into 3 types including the cloud, edge and IoT devices. More specifically, the first 10 servers, the last server, and the other 4 servers belong to IoT devices, the cloud, and the edge, respectively. Table 7 shows the detailed configurations and energy consumption of the servers [25]. The number of DNNs executed by an IoT device is regarded as the workload, is set to one in RQ1, RQ3 and RQ4, and ranges from 1 to 5 in RQ2. Besides, we assume that each IoT device can only connect to two nearby edge servers, and the bandwidths between different servers are shown in Table 4. During a period of time, the cloud-edge environment and the workload remain static, based on which the offloading decision is made.

1. https://github.com/SPSO-GA/dataset
To find all possible deployment units during the layer partition process, the most ideal offloading environment in our experimental environments (i.e. from an IoT device to the edge server $s_{14}$ ) is selected with the consideration of server performance and data transmission rate to conduct the simulation offloading experiment of Algorithm 1. $\lambda$ and $v_{p}$ are set to 3 and $10 \mathrm{MB} \cdot \mathrm{s}^{-1}$, respectively.

In addition, the parameters of SPSO-GA were initialized by following [38], where $\Gamma=50, \Theta=300, w_{u}=0.8, w_{d}=0.2$, $c_{1}^{s}=0.9, c_{1}^{e}=0.2, c_{2}^{s}=0.4$, and $c_{2}^{e}=0.9$. Simulation experiments were conducted on the Win10 64-bit operating system with an i7-4790 $3.60 \mathrm{GHz}$ Intel(R) Core(TM) processor and 32GB RAM.

\subsection{Classic Methods}

GA [29] and Greedy [26] are introduced to make comparisons and evaluate the performance of the proposed SPSOGA for DNN-based smart IoT systems in the cloud-edge environments.

Greedy only considers edge servers and IoT devices but ignores the servers in the cloud. The algorithm offloads each layer to the servers with the lowest computing energy consumption within their deadlines. If a layer fails to satisfy the deadline constraint, it will be offloaded to the next cheapest server.

GA uses a binary problem encoding method, whose dimension is equal to the number of servers. Meanwhile, its fitness function is defined based on Eqs. (16)-(18). Thus, the offloading corresponding to an encoded chromosome also considers the runtime energy consumption, switching, and computing energy consumption of each server.

To verify the efficiency of layer partition, SPSO-GA without layer partition (i.e. PSO-GA) is also used as the comparison. GA, PSO-GA, and SPSO-GA belong to the evolutionary algorithms. In the experiments, these algorithms are considered convergent if they maintain the same global best particle in 50 continuous iterations. The offloading results may be different with the same configurations in an experiment. Therefore, the system energy consumption is measured by the average of 50 repeated experiments.

\subsection{RQ1. SPSO-GA with different deadline constraints}

Fig. 7 depicts the system energy consumption of different offloading strategies for one execution of DNN per IoT device with different deadline constraints. In general, the system energy consumption decreases as the deadline becomes looser by using SPSO-GA, PSO-GA, and GA. This is because the strategies based on meta-heuristic algorithms tend to allocate more layers to the energy-efficient servers when the deadline is not strict. Moreover, SPSO-GA and PSO-GA can achieve the same performance with different deadline constraints. The results also show that the system energy consumption for each type of DNNs is different. This is because they consist of different DNN layers with various data transmissions between layers. For example, the number of layers in AlexNet is more than the others, and thus more computing energy consumption is consumed by AlexNet. For GoogleNet, the computing energy consumption is less than that of other DNNs. This is because there are more layers that can be executed in parallel, and thus more layers 


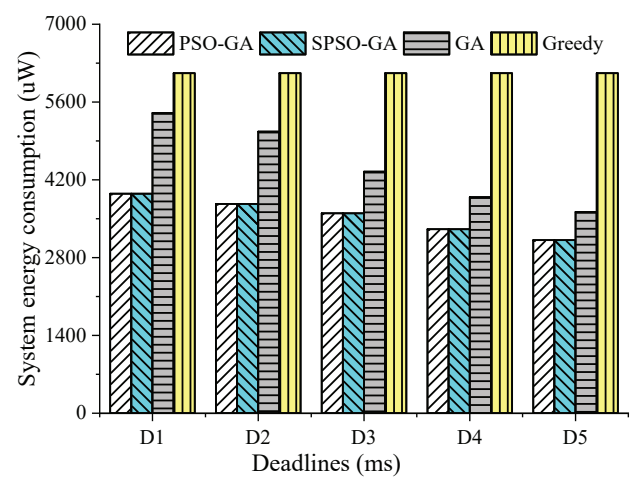

(a) AlexNet.

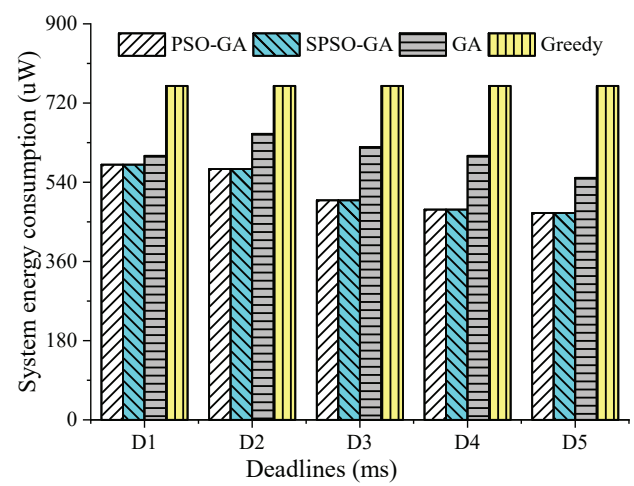

(c) GoogleNet.

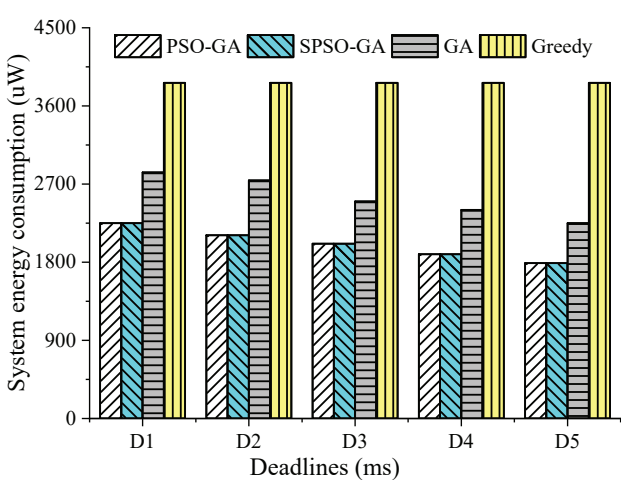

(b) VGG19.

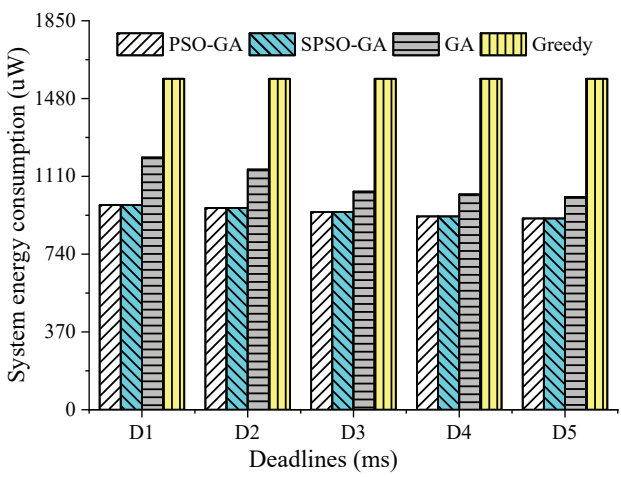

(d) ResNet101

Fig. 7. System energy consumption of different offloading strategies for one execution of DNN per loT device with different deadline constraints.

can be executed on servers with less energy consumption within the same deadline.

SPSO-GA and PSO-GA can adaptively adjust the search ability according to the current situation and evolve iteratively from a global perspective, which both perform best for all types of DNNs. Greedy only considers IoT devices and edge servers when offloading layers. It is a fixed strategy and only goes one way until it fails to meet deadline constraints. Therefore, the Greedy can obtain a feasible solution for all types of DNNs and consume the same system energy consumption with different deadline constraints. By contrast, the performance of GA is greatly influenced by deadline constraints. This is because its search scope is local at each iteration. As for the proposed SPSOGA and PSO-GA, they can save around 12.91\% 20.97\% and $31.55 \%$ 47.98\% compared with GA and Greedy, respectively.

Fig. 7(d) depicts the system energy consumption of different strategies for one execution of ResNet101 per IoT device, which is less than that in Figs. 7(a) and 7(b). This is because the calculation amount of layers in ResNet101 is less than that of AlexNet and VGG19 and thus leads to less computing energy consumption. In Fig. 7(c), the performance of SPSO-GA is a little better than GA with $D_{1}$. By using SPSO-GA, the layers can be offloaded to suitable servers from a global perspective even with strict deadline constraints.

\subsection{RQ2. SPSO-GA with different workloads}

Fig. 8 depicts the system energy consumption of different offloading strategies for different numbers of executing DNN per IoT device with a fixed deadline constraint (i.e. $D_{3}$ ). The bars depict the system energy consumption of different offloading strategies with various workloads, while the curves depict the energy saving rate $R_{e}\left(\mathrm{G}_{i}\right) . R_{e}\left(\mathrm{G}_{i}\right)=$ $-\left(e_{t}\left(\mathrm{~S}_{\mathrm{p}}\right)-e_{t}\left(\mathrm{G}_{i}\right)\right) / e_{t}\left(\mathrm{G}_{i}\right), \mathrm{G}_{i}=\left\{\mathrm{G}_{\mathrm{a}}, \mathrm{G}_{\mathrm{r}}\right\}$, where $\mathrm{S}_{\mathrm{p}}, \mathrm{G}_{\mathrm{a}}$, and $\mathrm{G}_{\mathrm{r}}$ are the offloading strategies based on PSO-GA, $\mathrm{GA}$, and Greedy, respectively. $e_{t}(\mathrm{~A})$ is the system energy consumption by using the algorithm A. Generally, SPSO-GA and PSO-GA achieve better offloading performance than the other two strategies. The system energy consumption of different offloading strategies increases as the workloads become heavier. This is because more servers are needed to execute the increasing DNN layers, which results in more system energy consumption.

As shown in Fig. 8, the system energy consumption of different offloading strategies grows as the number of executing DNN per IoT device increases. This is because the offloading strategies need to handle more layers with more servers, which results in more system energy consumption. In general, the results of the system energy consumption in Fig. 8 are similar to that in Fig. 7. Moreover, DNN type significantly affects the system energy consumption of the offloading strategies. Specifically, as the number of executing DNN per IoT device increases, the energy saving rate by using GA and Greedy grows significantly for AlexNet, VGG19, and ResNet101 but not obviously for GoogleNet. 


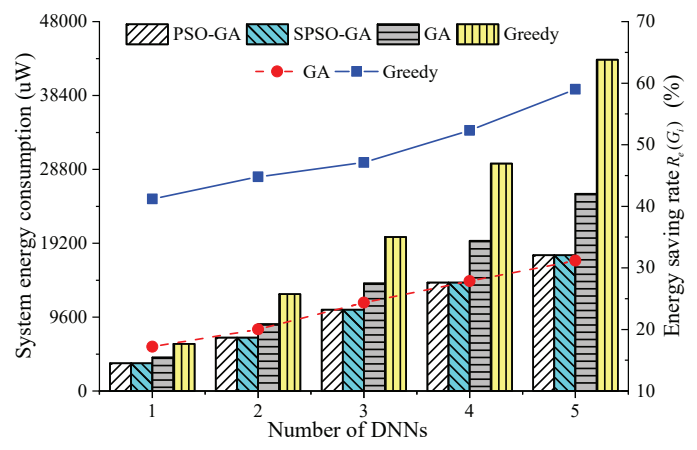

(a) AlexNet.

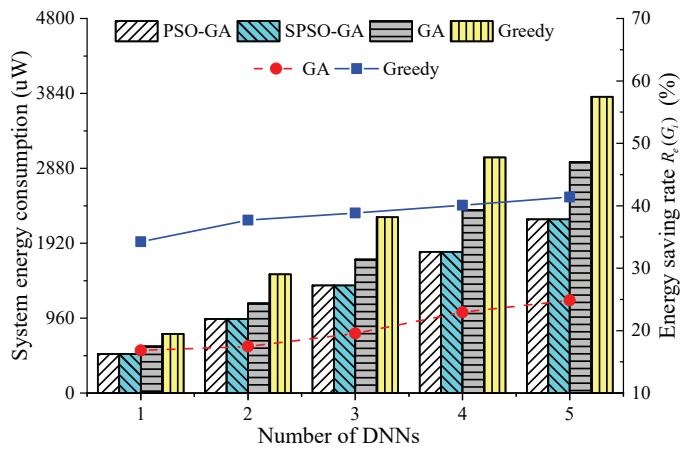

(c) GoogleNet.

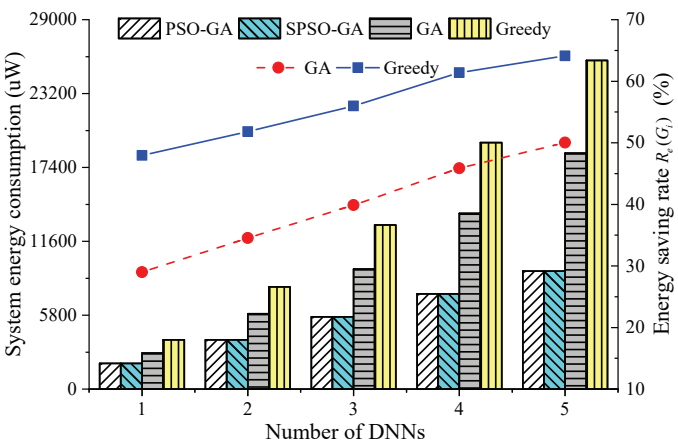

(b) VGG19.

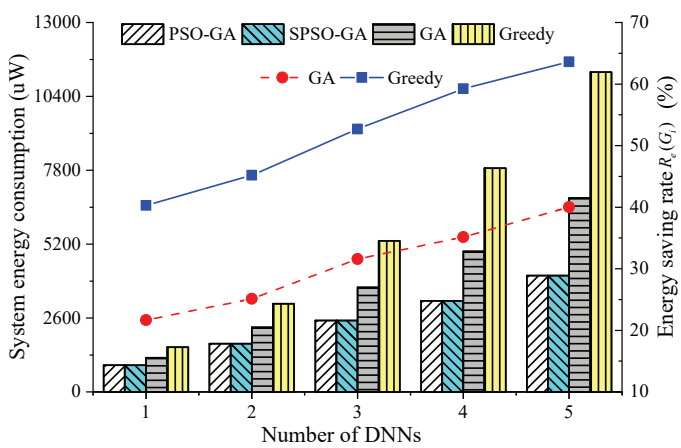

(d) ResNet101.

Fig. 8. System energy consumption of different offloading strategies for different numbers of executing DNN per loT device with a fixed deadline constraint.

In addition, the parallel structure of GoogleNet causes the growth rate of the system energy consumption by using PSO-GA and SPSO-GA to be almost the same as that by using GA and Greedy. By contrast, the serial structures of AlexNet, VGG19, and ResNet101 make the performance advantages more obvious by using PSO-GA and SPSOGA. As for the proposed SPSO-GA, they can save around $21.17 \% \sim 36.52 \%$ and $40.92 \% \sim 57.04 \%$ compared with GA and Greedy, respectively.

\subsection{RQ3. Impact of varying the MEC resource supply}

Fig. 9 depicts the system energy consumption of different offloading strategies for one execution of AlexNet per IoT device at $D_{3}$ with varying MEC resources. The impact of varying supplies of MEC resources on offloading results is analyzed from two aspects: the computational capacities of edge servers and the number of edge servers.

Fig. 9(a) shows the system energy consumption of different offloading strategies for one execution of AlexNet per IoT device at $D_{3}$ with varying computational capacities of edge servers. The scaling ratios of computational capacities for 4 edge servers are set to $\{0.6,0.8,1,1.2,1.4\}$. When the scaling ratio is set to 1 , the offloading results are the same as that at $D_{3}$ in Fig. 7(a). In general, the system energy consumption decreases as the computational capacities of edge servers become stronger. This is because the offloading strategies tend to allocate more DNN layers to the edge rather than the cloud when edge servers are equipped with more computational resources, which leads to the reduction of energy consumption in cloud servers. Both SPSO-GA and PSO-GA perform best for all kinds of edge servers with different computational capacities. Specifically, they can reduce the system energy consumption by $15.43 \% \sim 17.88 \%$ compared with the GA. Similarly, SPSO-GA and PSO-GA can reduce the system energy consumption by about $28 \%$ compared with the Greedy.

Fig. 9(b) shows the system energy consumption of different offloading strategies for one execution of AlexNet per IoT device at $D_{3}$ with varying numbers of edge servers, which are set to $\{2,4,6,8,10\}$. The other configurations are kept the same as in Subsection 5.1. The detailed information about the configurations of edge servers can be found in our GitHub project. In general, the system energy consumption decreases as the number of edge servers increases. When the number of edge servers is small, the offloading strategies tend to deploy more DNN layers in the cloud for satisfying deadline constraints. This results in more system energy consumption. Otherwise, the offloading strategies tend to deploy more DNN layers in the edge rather than the cloud, which leads to less system energy consumption. Specially, both SPSO-GA and PSO-GA perform best for different numbers of edge servers. The performance of the GA is better than that of the Greedy.

\subsection{RQ4. Execution time of SPSO-GA and PSO-GA}

The results of Sections 5.3 and 5.4 indicate that the layer partition operations have seldom negative impact on the proposed SPSO-GA for optimizing the system energy consumption. The layer partition is able to lessen the number of deployment units in a DNN and thus improve the execution time of the proposed algorithm. Fig. 10 depicts the execution time of SPSO-GA and PSO-GA with different workloads. 


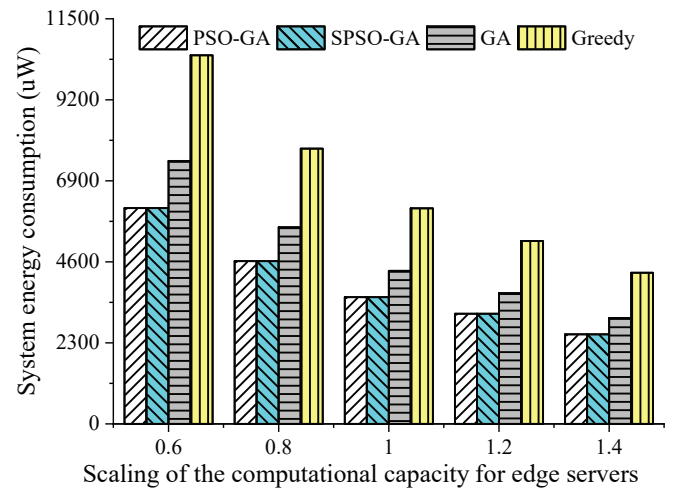

(a) Varying the computational capacity of edge servers.

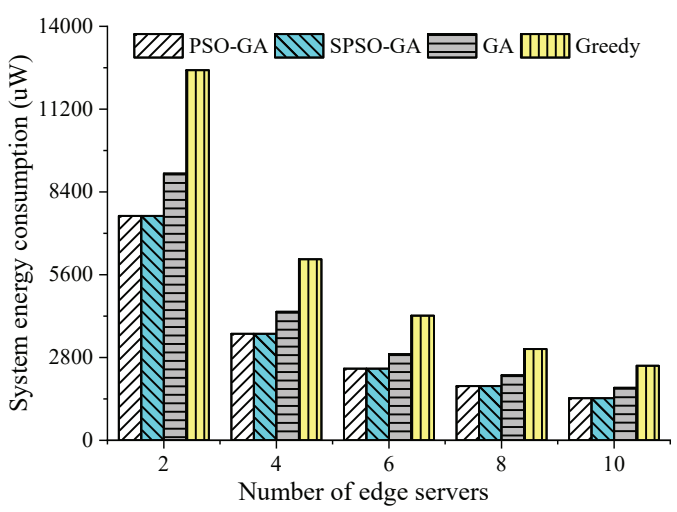

(b) Varying the number of edge servers.

Fig. 9. System energy consumption of different offloading strategies for one execution of AlexNet per loT device at $D_{3}$ with varying MEC resource.

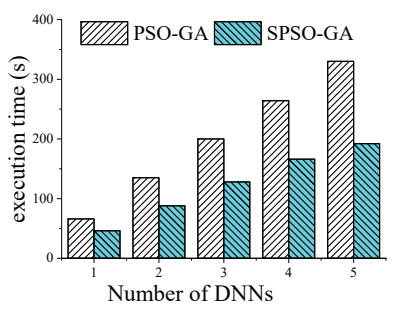

(a) AlexNet.

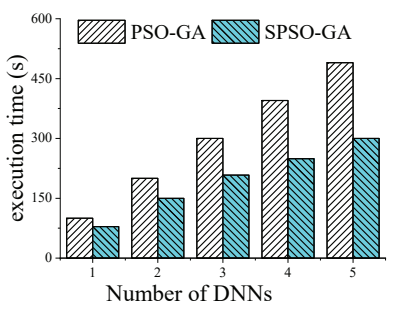

(b) VGG19.

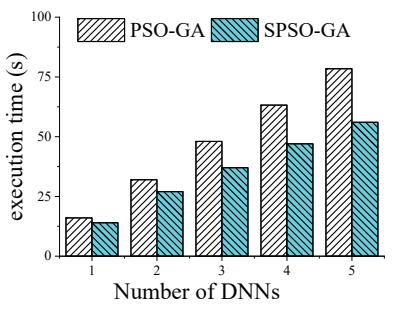

(c) GoogleNet.

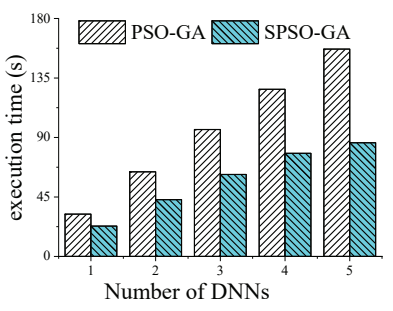

(d) ResNet101.

Fig. 10. Execution time of SPSO-GA and PSO-GA with different workloads.

There are different numbers of executing DNN per IoT device during a period of time, whose value is between 1 to 5 . In general, the execution time of SPSO-GA is much less than the PSO-GA on AlexNet, VGG19, and ResNet101, whose structures are serial. This is because DNNs with serial structures have more dimensionality reductions, which results in less deployment units after layer partition. The number of deployment units determines the problem encoding space and the execution time of the algorithms. Therefore, the execution time of SPSO-GA is much less than PSO-GA on AlexNet, VGG19, and ResNet101.

Fig. 10(a) depicts the execution time of SPSO-GA and PSO-GA with different workloads for AlexNet. Specifically, the execution time of SPSO-GA is around $36.01 \%$ less than PSO-GA on average. As shown in Figs. 10(b) to 10(d), the execution time of SPSO-GA is around $30.48 \%, 21.05 \%$, and $35.96 \%$ less than PSO-GA on average for VGG19, GoogleNet, and ResNet101, respectively. After layer partition, GoogleNet still remains many deployment units, and thus the execution time of SPSO-GA cannot be well reduced.

\section{Discussion}

Some issues about applicability are discussed as follows.

Impact of transmission speed on the performance. Data transmission speed is positively-correlated with bandwidth. When the bandwidth between IoT devices and the cloud increases, IoT devices tend to offload more DNN layers to the cloud. Due to the increase of data transmission speed, the latency of data transmission between IoT devices and the cloud decreases, which would better satisfy the deadline constraints for the DNN-based Smart IoT systems. Similarly, the increase of bandwidth between IoT devices and the edge has positive impact on the offloading results for the DNNbased Smart IoT systems.

Time complexity. In each iteration of the proposed SPSO-GA algorithm, all particles are updated and their fitness is calculated. The number of calculations required to update the state of particles is determined by the initial population size $\Gamma$ and the particle dimension $D$. The total number of deployment units in DNNs $y$ determines the complexity of the fitness function based on Algorithm 2. Since $D=y$ in the proposed offloading strategy, the time complexity of SPSO-GA is $O\left(\Gamma \cdot y^{2}\right)$ per iteration. In addition, the convergence time is influenced by the total number of deployment units in DNNs $y$ and the number of servers $|\boldsymbol{C}|$. The overall framework of the PSO-GA and GA is the same as SPSO-GA, and thus the time complexity of the PSO-GA and GA should be $O\left(\Gamma \cdot y^{2}\right)$ per iteration. Note that $y$ in PSOGA and GA is equal to the number of DNN layers before layer partition operations. Obviously, the time complexity of the Greedy is $O(y \cdot|\boldsymbol{C}|)$.

\section{CONCLUSION}

In this paper, we have proposed an energy-efficient offloading strategy based on SPSO-GA for DNN-based smart IoT systems in the cloud-edge environments, which aims to reduce the system energy consumption while satisfying the deadline constraints. The extensive simulation experiments show that the proposed strategy can achieve the superior performance than other classic methods. The system energy 
consumption is reduced as the deadline becomes looser by using SPSO-GA, PSO-GA, and GA. With the looser deadline constraints, more layers tend to be allocated to the more energy-efficient servers in the same situation. However, the performance of GA is greatly influenced by the deadline constraints due to its local search scope at each iteration.

In our future work, we will consider more complicated scenarios with the environmental fluctuation including network delay, bandwidth fluctuation, and server failure, and investigate the adaptiveness of the proposed offloading strategy. Moreover, we plan to apply deep reinforcement learning algorithm to offloading decision on DNN-based smart IoT systems.

\section{ACKNOWLEDGMENTS}

This work is partly supported by the Natural Science Foundation of China under Grant No. 62072108, the Natural Science Foundation of Fujian Province for Distinguished Young Scholar No. 2020J06014, the Natural Science Foundation of Fujian Province under Grant No. 2019J01286, and the Young and Middle-aged Teacher Education Foundation of Fujian Province under Grant No. JT180098.

\section{REFERENCES}

[1] H. Li, K. Ota, and M. Dong, "Learning IoT in edge: Deep learning for the internet of things with edge computing," IEEE Network, vol. 32, no. 1, pp. 96-101, 2018.

[2] Y. You, Z. Zhang, C. Hsieh, J. Demmel, and K. Keutzer, "Fast deep neural network training on distributed systems and cloud TPUs," IEEE Transactions on Parallel and Distributed Systems, vol. 30, no. 11, pp. 2449-2462, 2019.

[3] C. Hu, W. Bao, D. Wang, and F. Liu, "Dynamic adaptive DNN surgery for inference acceleration on the edge," in IEEE International Conference on Computer Communications (INFOCOM), 2019.

[4] Y. Kang, J. Hauswald, C. Gao, A. Rovinski, T. Mudge, J. Mars, and L. Tang, "Neurosurgeon: Collaborative intelligence between the cloud and mobile edge," in the 22nd International Conference on Architectural Support for Programming Languages and Operating Systems, 2017.

[5] H. Badri, T. Bahreini, D. Grosu, and K. Yang, "Energy-aware application placement in mobile edge computing: A stochastic optimization approach," IEEE Transactions on Parallel and Distributed Systems, vol. 31, no. 4, pp. 909-922, 2020.

[6] B. Lin, Y. Huang, J. Zhang, J. Hu, X. Chen, and J. Li, “Cost-driven off-loading for DNN-based applications over cloud, edge, and end devices," IEEE Transactions on Industrial Informatics, vol. 16, no. 8, pp. 5456-5466, 2020.

[7] M. Chen, S. Guo, K. Liu, X. Liao, and B. Xiao, "Robust computation offloading and resource scheduling in cloudlet-based mobile cloud computing," IEEE Transactions on Mobile Computing, vol. 20, no. 5, pp. 2025-2040, 2021.

[8] M. Altamimi, A. Abdrabou, K. Naik, and A. Nayak, "Energy cost models of smartphones for task offloading to the cloud," IEEE Transactions on Emerging Topics in Computing, vol. 3, no. 3, pp. 384398, 2015.

[9] K. Elgazzar, P. Martin, and H. S. Hassanein, "Cloud-assisted computation offloading to support mobile services," IEEE Transactions on Cloud Computing, vol. 4, no. 3, pp. 279-292, 2016.

[10] Z. Fang, T. Yu, O. J. Mengshoel, and R. K. Gupta, "Qos-aware scheduling of heterogeneous servers for inference in deep neural networks," in International Conference on Information and Knowledge Management (ICIKM), 2017.

[11] K. Kumar and Y. Lu, "Cloud computing for mobile users: Can offloading computation save energy?" Computer, vol. 43, no. 4, pp. 51-56, 2010.

[12] H. J. Jeong, "Lightweight offloading system for mobile edge computing," in IEEE International Conference on Pervasive Computing and Communications Workshops (ICPCCW), 2019.
[13] M. Chen and Y. Hao, "Task offloading for mobile edge computing in software defined ultra-dense network," IEEE Journal on Selected Areas in Communications, vol. 36, no. 3, pp. 587-597, 2018.

[14] X. Chen, L. Jiao, W. Li, and X. Fu, "Efficient multi-user computation offloading for mobile-edge cloud computing," IEEE/ACM Transactions on Networking, vol. 24, no. 5, pp. 2795-2808, 2016.

[15] Z. Ali, L. Jiao, T. Baker, G. Abbas, Z. H. Abbas, and S. Khaf, "A deep learning approach for energy efficient computational offloading in mobile edge computing," IEEE Access, vol. 7, pp. 149623-149633, 2019.

[16] C. Lo, Y. Y. Su, C. Y. Lee, and S. C. Chang, "A dynamic deep neural network design for efficient workload allocation in edge computing," in IEEE International Conference on Computer Design (ICCD), 2017.

[17] H. J. Jeong, I. Jeong, H. J. Lee, and S. M. Moon, "Computation offloading for machine learning web apps in the edge server environment," in International Conference on Distributed Computing Systems (ICDCS), 2018.

[18] L. Yang, J. Cao, S. Tang, D. Han, and N. Suri, "Run time application repartitioning in dynamic mobile cloud environments," IEEE Transactions on Cloud Computing, vol. 4, no. 3, pp. 336-348, 2016.

[19] T. Mohammed, C. Joe-Wong, R. Babbar, and M. D. Francesco, "Distributed inference acceleration with adaptive DNN partitioning and offloading," in IEEE International Conference on Computer Communications (INFOCOM), 2020.

[20] H. Wu, W. J. Knottenbelt, and K. Wolter, "An efficient application partitioning algorithm in mobile environments," IEEE Transactions on Parallel and Distributed Systems, vol. 30, no. 7, pp. 1464-1480, 2019.

[21] S. Teerapittayanon, B. McDanel, and H. Kung, "Distributed deep neural networks over the cloud, the edge and end devices," in International Conference on Distributed Computing Systems (ICDCS), 2017.

[22] H. Lin, S. Zeadally, Z. Chen, H. Labiod, and L. Wang, "A survey on computation offloading modeling for edge computing," Journal of Network and Computer Applications, vol. 169, pp. 1-25, 2020.

[23] C. Hu, W. Bao, D. Wang, and F. Liu, "Dynamic adaptive DNN surgery for inference acceleration on the edge," in IEEE International Conference on Computer Communications (INFOCOM), 2019.

[24] N. Zhao, X. Liu, F. R. Yu, M. Li, and V. C. M. Leung, "Communications, caching, and computing oriented small cell networks with interference alignment," IEEE Communications Magazine, vol. 54, pp. 29-35, 2016.

[25] G. Xie, G. Zeng, X. Xiao, R. Li, and K. Li, "Energy-efficient scheduling algorithms for real-time parallel applications on heterogeneous distributed embedded systems," IEEE Transactions on Parallel and Distributed Systems, vol. 28, no. 12, pp. 3426-3442, 2017.

[26] C. Jiang, Z. Chen, R. Su, and Y. C. Soh, "Group greedy method for sensor placement," IEEE Transactions on Signal Processing, vol. 67, no. 9, pp. 2249-2262, 2019.

[27] D. S. Hochba, "Approximation algorithms for NP-hard problems," SIGACT News, vol. 28, pp. 40-52, 1997.

[28] B. Lin, F. Zhu, J. Zhang, J. Chen, X. Chen, N. N. Xiong, and J. Lloret Mauri, "A time-driven data placement strategy for a scientific workflow combining edge computing and cloud computing," IEEE Transactions on Industrial Informatics, vol. 15, no. 7, pp. 42544265, 2019.

[29] L. Cui, J. Zhang, L. Yue, Y. Shi, H. Li, and D. Yuan, "A genetic algorithm based data replica placement strategy for scientific applications in clouds," IEEE Transactions on Services Computing, vol. 11, no. 4, pp. 727-739, 2018.

[30] J. S. Su, W. Z. Guo, C. L. Yu, and G. L. Chen, "Fault-tolerance clustering algorithm with load-balance aware in wireless sensor network," Jisuanji Xuebao/Chinese Journal of Computers, vol. 37, pp. 445-456, 2014.

[31] M. A. Rodriguez and R. Buyya, "Deadline based resource provisioningand scheduling algorithm for scientific workflows on clouds," IEEE Transactions on Cloud Computing, vol. 2, no. 2, pp. 222-235, 2014.

[32] H. Li, D. Yang, W. Su, J. LÃij, and X. Yu, "An overall distribution particle swarm optimization MPPT algorithm for photovoltaic system under partial shading," IEEE Transactions on Industrial Electronics, vol. 66, no. 1, pp. 265-275, 2019.

[33] D. O'Neill, A. Lensen, B. Xue, and M. Zhang, "Particle swarm optimisation for feature selection and weighting in high-dimensional clustering," in IEEE Congress on Evolutionary Computation (ICEC), 2018. 
[34] M. Masdari, F. Salehi, M. Jalali, and M. Bidaki, "A survey of PSO-based scheduling algorithms in cloud computing," Journal of Network and Systems Management, vol. 25, pp. 122-158, 2017.

[35] T. R. Chavan and A. V. Nandedkar, "A hybrid deep neural network for online learning," in the 9th International Conference on Advances in Pattern Recognition (ICAPR), 2017.

[36] X. Chen, M. Li, H. Zhong, Y. Ma, and C.-H. Hsu, "DNNOff: Offloading dnn-based intelligent iot applications in mobile edge computing," IEEE Transactions on Industrial Informatics, pp. 1-1, 2021.

[37] H. Topcuoglu, S. Hariri, and Min-You Wu, "Performance-effective and low-complexity task scheduling for heterogeneous computing," IEEE Transactions on Parallel and Distributed Systems, vol. 13, no. 3, pp. 260-274, 2002.

[38] Y. Shi and R. Eberhart, "A modified particle swarm optimizer," in IEEE International Conference on Evolutionary Computation Proceedings (ICECP), 1998.

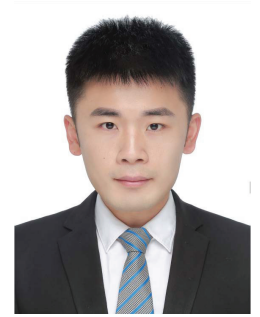

Zheyi Chen is currently a Ph.D. candidate in computer science at the University of Exeter. $\mathrm{He}$ received his M.Sc. degree in Computer Science from Tsinghua University, China, in 2017, and B.Sc. degree in Computer Science from Shanxi University, China, in 2014. His research interests include cloud computing, mobile edge computing, deep learning, and resource optimization.

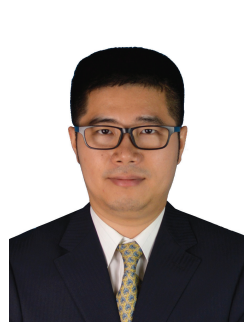

Xing Chen received the B.S. degree and the Ph.D. degree from Peking University, in 2008 and 2013, respectively. Upon completion of the Ph.D. degree, he joined Fuzhou University and has held the rank of Professor since 2020. Now he is the deputy director of Fujian Provincial Key Laboratory of Network Computing and Intelligent Information Processing (Fuzhou University), and leads the Systems research group. Dr. Chen's research focuses on the software systems and engineering approaches for cloud and mobility. His current projects cover the topics from self-adaptive software, computation offloading, model driven approach and so on. He has published over fifty journal and conference articles, and was awarded two First Class Prizes for Provincial Scientific and Technological Progress, separately in 2018 and 2020.

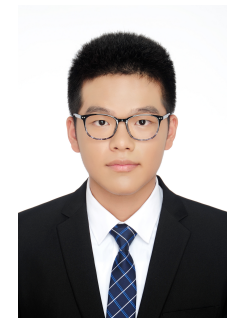

Jianshan Zhang received his M.S. degree in Materials Engineering from Fujian Normal University, China, in 2018. He is currently working toward the $\mathrm{PhD}$ degree at the College of Mathematics and Computer Science, Fuzhou University. He has also been a part of the Fujian Key Laboratory of Network Computing and Intelligent Information Processing at Fuzhou University since September 2019. His current research interests include edge computing, computational intelligence and cloud computing.

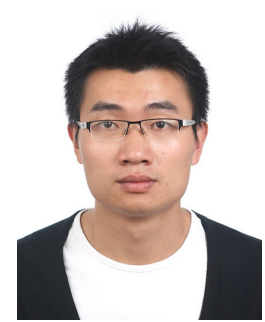

Bing Lin received the B.S. and M.S degrees in Computer Science from Fuzhou University, Fuzhou, China, in 2010 and 2013, respectively, and the Ph.D. degree in Communication and Information System from Fuzhou University in 2016. He is currently an associate professor with the College of Physics and Energy at Fujian Normal University. Now he is the deputy director of the Department of Energy and Materials, and leads the Intelligent Computing research group. His research interest mainly includes parallel and distributed computing, computational intelligence, and data center resource management. He has published over twenty journals and conference articles, such as IEEE Transactions on Industrial Informatics, and IEEE Transactions on Network and Service Management.

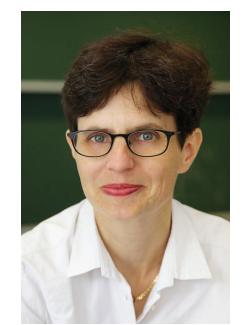

Katinka Wolter received her Ph.D degree from Technische Universität Berlin in 1999. She has been Assistant professor at Humboldt-University Berlin and lecturer at Newcastle University before joining Freie Universität Berlin as a professor for dependable systems in 2012. Her research interests are model-based evaluation and improvement of dependability, security and performance of distributed systems and networks. Special systems of interest to her are cryptocurrencies, data streaming systems and car traffic

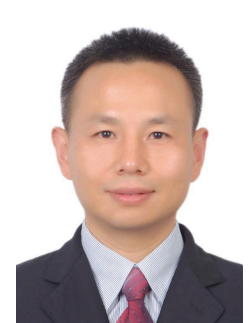

Geyong Min is a Professor of High Performance Computing and Networking in the Department of Computer Science within the College of Engineering, Mathematics and Physical Sciences at the University of Exeter, United Kingdom. He received the Ph.D. degree in Computing Science from the University of Glasgow, United Kingdom, in 2003, and the B.Sc. degree in Computer Science from Huazhong University of Science and Technology, China, in 1995. His research interests include Computer Networks, Wireless Communications, Parallel and Distributed Computing, Ubiquitous Computing, Multimedia Systems, Modelling and Performance Engineering. 